${ }^{*}$ Graphical Abstract

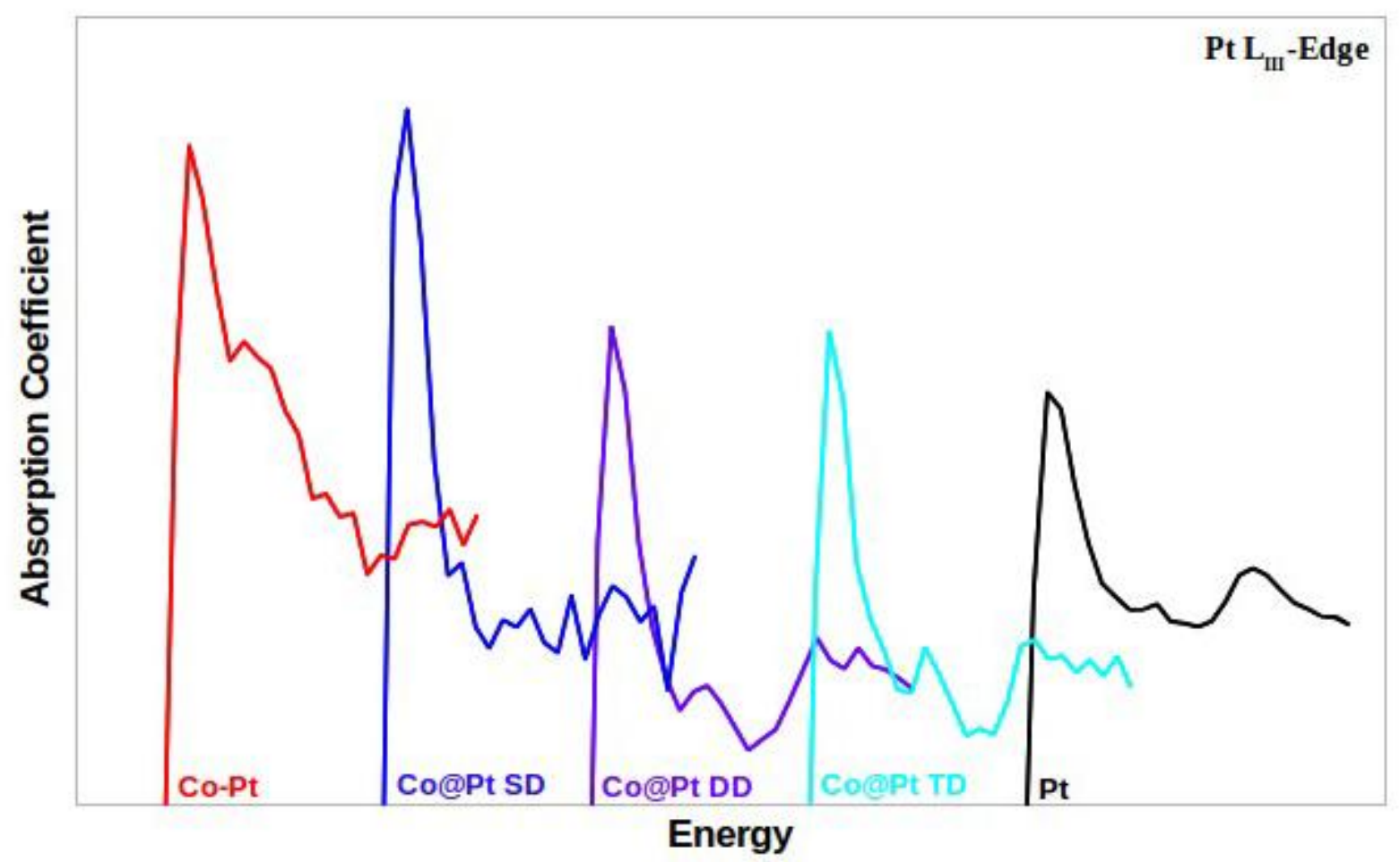




\title{
Characterization of Ni@Pt and Co@Pt Overlayer Catalysts Using XAS Studies
}

Allen R. Morris, Michael D. Skoglund, Joseph H. Holles*

\author{
Department of Chemical and Petroleum Engineering \\ University of Wyoming
}

Dept. 3295

1000 E. University Ave.

Laramie, WY - 82071

\begin{abstract}
* To whom correspondence should be addressed
jholles@uwyo.edu

(307) 766-6772

Fax: (307) 766-2221
\end{abstract}




\begin{abstract}
The use of XAS studies on Co@Pt and Ni@Pt pseudomorphic overlayer catalysts along with a ethylene hydrogenation descriptor reaction have shown that the d-band of Pt can be effected by the parent metal that it is overlayed on. Pt bond distances were found to be shorter than pure Pt when atop the base $\mathrm{Ni}$ and $\mathrm{Co}$ indicating that the base metal was exerting compressive strain on the Pt and, for two reasons, causing the $\mathrm{Pt} d$-band to broaden and therefore shift downwards in energy. First, the compressive strain alone has been shown to broaden the d-band of larger atoms atop of smaller ones (i.e. Pt atop $\mathrm{Ni}$ ) and in the case of the Co@Pt sample Co has one less valence electron then $\mathrm{Ni}$ and $\mathrm{Pt}$. This allows for more, than $\mathrm{Ni}$ and $\mathrm{Pt}$, of a synergistic interaction between $\mathrm{Co}$ and $\mathrm{Pt}$ in the overlayer samples resulting in even larger Pt d-bandwidth broadening and shifting. This XAS data was confirmed via reactivity studies as the overlayer samples of both $\mathrm{Ni}$ and $\mathrm{Co}$ based catalysts had lower reactivity than the pure Pt samples.
\end{abstract}




\section{Introduction:}

Bimetallic catalysts play an important role in many catalytic processes. It has been demonstrated that various metal combinations can improve activity, selectivity, and catalyst life within certain systems [1-5]. Several different mechanisms have been proposed to explain the changes in catalytic behavior seen with bimetallic systems. One theory involves structural effects (interatomic distance and coordination number) [6]; another proposes electronic modification of surface properties (electron withdrawing or donating) [7]. Single crystal and computational studies have been conducted to further understand electronic modifications seen in bimetallic systems and have found that the creation of a pseudomorphic overlayer catalyst (a monatomic layer of one metal atop a different base bulk metal) could result in the creation of an improved catalyst for a wide variety of reaction systems [8-17]. Through pairing different metals in an overlayer system, properties such as $\mathrm{H}_{2}$ adsorption strength (or other adsorbates) can be altered when compared to their monometallic counterparts. Christoffersen et al. have computationally determined that the creation of an overlayer system can change the center of the d-band of the overlaying metal, ultimately resulting in a change in bonding strength of adsorbates on the catalyst [18]. Through pairing certain overlayer and base metal combinations, stronger or weaker adsorbate bonds can be achieved as desired for the reaction of interest.

In previous work, our laboratory has used the directed deposition technique to create Re@Pd, $\mathrm{Ni@Pt,} \mathrm{and} \mathrm{Co@Pt} \mathrm{(base@overlayer)} \mathrm{overlayer} \mathrm{bimetallic} \mathrm{catalysts} \mathrm{[19-21]} \mathrm{in} \mathrm{an} \mathrm{effort} \mathrm{to} \mathrm{create}$ supported catalysts which manifest the properties predicted for pseudomorphic overlayers. The directed deposition technique makes use of a selective surface reaction to preferentially deposit an overlayer metal only atop existing host metal sites while the support surface remains deactivated so that the overlayer metal is not separately deposited on the support [22]. Hydrogen and carbon monoxide chemisorption and ethylene hydrogenation descriptor studies have been conducted on $\mathrm{Ni@Pt}$ and Co@Pt overlayer catalysts [20]. It was found that when compared to platinum only catalysts, catalysts with platinum deposited using a directed deposition technique atop a $\mathrm{Ni}$ or Co base metal particle, showed a decrease in hydrogen bond strength and ethylene hydrogenation activity. The decrease in hydrogen binding strength is consistent with the computational predictions and results in a decreased surface coverage of hydrogen which in-turn results in an activity decrease. Also, comparison between 
overlayer and bimetallic catalysts of similar loading resulted in confirmation that the directed deposition synthesis technique was not merely producing non-structured bimetallic catalysts.

Structural knowledge of the above overlayer and non-structured bimetallic catalysts is necessary in order to fully understand the structure/property relationship and improve their catalytic activity. To further characterize Ni@Pt and Co@Pt overlayer catalysts, X-ray absorption spectroscopy (XAS) studies have been conducted. XAS is capable of determining element specific atomic structural properties (coordination number, bond distance, oxidation states, etc) [23-25]. XAS studies allow for the investigation of small, highly disperse, metal particles; something many other structural characterization techniques are incapable of examining. Extended X-ray absorption fine structure (EXAFS) spectroscopy probes the immediate atomic surroundings of an absorber atom. EXAFS studies on overlayer catalysts should be capable of examining the atomic neighborhood of a deposited overlayer metal and determining if it exists in a thin film as desired, bulk clusters, or other structures on the base metal particle. In addition to the structural parameters gathered via EXAFS, X-ray Absorption Near Edge Structure (XANES) analysis will be used to look at the electronic phenomenon exhibited by the overlayer and base metals of the catalyst systems. XANES, and specifically the white line height, gives an indication of the amount of interaction between the parent metal (Co or $\mathrm{Ni}$ ) and the overlayer metal $(\mathrm{Pt})$. This paper will examine the use of XAS and FEFF modeling to further characterize Ni@Pt and Co@Pt overlayer catalysts and compare them to their bimetallic counterparts.

\section{Experimental:}

\subsection{Catalyst Synthesis}

The monometallic parent catalysts and bimetallic catalysts used for this paper were created using standard techniques [20]. All catalysts were supported with a $\gamma$-alumina (Alfa Aesar, 99.97\%).

The pseudomorphic overlayer catalysts used in this paper were prepared using the directed deposition technique. The parent $\mathrm{Ni}$ or $\mathrm{Co} / \mathrm{Al}_{2} \mathrm{O}_{3}$ base catalyst was reduced in $60 \mathrm{sccm} \mathrm{H}_{2}$ flow at $450^{\circ} \mathrm{C}$ for 4 hours using a $3^{\circ} \mathrm{C} / \mathrm{min}$ ramp rate. The catalyst was then cooled to room temperature and water- 
saturated helium gas was passed over the catalyst for 24 hours. The nickel catalyst was then briefly reduced at $60^{\circ} \mathrm{C}$ in $60 \mathrm{sccm} \mathrm{H}_{2}$ flow for 1 hour. The cobalt catalyst was reduced at the same flow rate but at $75^{\circ} \mathrm{C}$. These reduction temperatures were found to be where each parent catalyst had maximum irreversible $\mathrm{H}_{2}$ adsorption. The reduced catalyst was then quickly added to a solution of acetylacetone, used as a surface inhibitor to ensure low platinum adsorption on the support, and toluene at room temperature. The surface deactivation was allowed to proceed for 15 minutes before a solution of Pt (II) acetylacetonate and toluene was added. The reaction was allowed to proceed for 2 hours at $60^{\circ} \mathrm{C}$. The catalyst was then vacuum filtered to remove liquid and allowed to dry in the fume hood. The sample was then placed in a $110^{\circ} \mathrm{C}$ drying oven overnight to fully dry the catalyst. The catalyst was then loaded into a quartz tube to be calcined in near stagnant air at $320^{\circ} \mathrm{C}$ for $\mathrm{Ni} @ \mathrm{Pt}$ (to prevent alloying [10]) and $400^{\circ} \mathrm{C}$ for Co@Pt for 4 hours using a $3^{\circ} \mathrm{C} / \mathrm{min}$ ramp rate up and down. After calcination the catalyst was reduced in $10 \mathrm{sccm} \mathrm{H}_{2}$ flow at calcine temperatures for 4 hours using a $3^{\circ} \mathrm{C} / \mathrm{min}$ ramp rate. Multiple overlayer depositions were done by repeating the above procedure to produce single, double, and triple deposition $\mathrm{Ni@Pt}$ and Co@Pt samples.

\subsection{Hydrogen Chemisorption Experiments}

Hydrogen chemisorption studies were conducted using a Micromeritics APAP 2020. Prior to analysis, samples were reduced at $400^{\circ} \mathrm{C}$ under flowing hydrogen. Isotherms were obtained at temperatures between $35^{\circ} \mathrm{C}$ and $300^{\circ} \mathrm{C}$ at pressures ranging from $1 \mathrm{mTorr}$ to 900 Torr. Metal dispersions were obtained through chemisorption results.

\subsection{Ethylene Hydrogenation Reactivity Experiments}

Reactivity studies were carried out using an ethylene hydrogenation descriptor reaction and is described in detail by Skoglund et al. [20]. This reaction was carried out with varying ethylene flows and at various temperatures, but the temperature study is the only one reported here.

\subsection{XAS Experiments}

Nickel and cobalt K-Edge $\left(E_{0}=8333 \mathrm{eV}\right.$ and $E_{0}=7709 \mathrm{eV}$, respectively) along with platinum $\mathrm{L}_{11 I^{-}}$ edge $\left(E_{0}=11564 \mathrm{eV}\right)$ X-ray adsorption spectroscopy $(\mathrm{XAS})$ experiments were conducted using the 
Materials Research Collaborative Access Team (MRCAT, 10-ID) beamline at the Advanced Photon Source (APS, 7.0 GeV ring energy), Argonne National Laboratory. A double-crystal Si(111) monochromator was used along with an uncoated glass mirror capable of minimizing harmonics. Nickel and cobalt data was collected in transmission mode. Due to the low platinum loadings (shown in Table 1) [20], platinum data was collected in fluorescence mode [25]. Transmission catalyst samples were pressed into a thin wafer inside a six sample, steel transmission sample holder which held the sample perpendicular to the incident X-ray beam. Fluorescence samples were pressed into a thin wafer inside a steel sample holder at a $45^{\circ}$ angle for sampling. Each fluorescence sample holder had one face left open in order to allow the X-rays to pass through to the detector after making contact with the angled catalyst surface. Catalyst sample holders were then loaded into an in-house designed continuous flow quartz reactor cell (18 in. long and 0.75 in. in diameter) with polyimide windows and valves capable of isolating samples from the atmosphere and analyzing samples in both fluorescence and transmission mode [26]. A cone off the side in the center of the reactor cell aligned with the hole in the sample holder allowed for fluorescence X-rays to reach the detector. Each sample was reduced under $4 \% \mathrm{H}_{2} / \mathrm{He}$ flow at $400^{\circ} \mathrm{C}$ for several hours before analysis. XAS sampling was done at room temperature under a helium atmosphere. Transmission measurements were done in quick scan mode using a gas ionization chamber as the detector and multiple data sets were obtained and averaged. Fluorescence measurements were conducted at an angle of $90^{\circ}$ to the incident X-ray beam using a cryogenically cooled 13-element Germanium detector. Fluorescence measurements were made using continuous scanning for a duration of $2 \mathrm{sec}$. per data point measured every $0.05 \mathrm{eV}$.

\subsection{Data Analysis}

Standard methods were used to extract the EXAFS data from the measured absorption spectra $[27,28]$. Multiple scans were collected for each sample and samples were normalized, aligned to the foil edge, and averaged/merged using Athena, part of the IFEFFIT/Demeter software package [29]. Fourier transformation (FT) of the EXAFS data allows for the isolation of primary scattering paths. Forward $\mathrm{k}^{2}$ weighted Fourier transform (FT) data ranges were determined by selecting the largest range of usable kspace data; 3.5-13 $\AA^{-1}$ for Ni edges, 2.5-11 $\AA^{-1}$ for Co edges, and 4.0-10.0 $\AA^{-1}$ for Pt edges. Inverse $k^{2}$ 
weighted transforms were performed over the non-phase corrected radial range of each primary metalmetal single scattering peak; 1.8-2.6 $\AA$ for $\mathrm{Ni}, 1.9-3.0 \AA$ for $\mathrm{Co}$, and 1.5-3.5 $\AA$ for Pt. All Fourier transforms were carried out using a Hanning window. Fitting analysis was completed on the Fourierfiltered data.

FEFF modeling and data fitting were completed using Artemis which is also part of the IFEFFIT/Demeter package. Crystal structure input files were obtained from the American Mineralogist Crystal Structure Database [30] to build FEFF models of each catalyst (F.C.C. Ni [31], H.C.P. Co [32], F.C.C Pt [33]) that were capable of modeling backscattering amplitude and phase. The first coordination shell of FEFF calculations were then fitted with the nickel and cobalt empirical data using the classic single-scattering approximation; i.e. multiple scattering paths were not included. The first two scattering shells were necessary for all platinum data in order to obtain a reasonable peak fit. Main peaks in the data were isolated and fitted using standard procedures [34]. In order to complete a fit of the first metalmetal scattering shell, the R-space region of interest was isolated and back-transformed. After the R range of interest was determined, a FEFF model was built to represent each crystal and scattering shell calculations were completed. The first scattering shell was used to fit Co and Ni K-edge data as the first shell was the only scattering shell to result in a peak at the desired R-range. In order to calculate coordination number $(\mathrm{N})$ it was first set at its expected value, 12 for all samples, so that the Debye-Waller factor $\left(\Delta \sigma^{2}\right)$, amplitude $\left(\delta_{0}{ }^{2}\right)$, and $\Delta \mathrm{E}_{0}$ could be calculated. After the initial fit, the Debye-Waller factor was set and $\mathrm{N}$ was changed to a floating variable; a second fit was then completed, allowing for the calculation of coordination number.

\section{Results}

\subsection{Elemental Analysis and Dispersions}

Elemental analysis was conducted by Galbraith Laboratories, Inc. in order to determine actual metal loadings for each catalyst. Hydrogen chemisorption was used to determine metal dispersions. The metal loadings and dispersions (\% metal atoms exposed) for each catalyst are reported in Table 1. For all but one of the bimetallic overlayer samples, each additional deposition resulted in an increase in Pt loading. The Co@Pt triple deposition (TD) was the only sample to not show an increase in Pt loading, 
perhaps indicating the limit of the synthesis procedure for Co@Pt systems. Non-structured bimetallic alloy catalysts were also synthesized with loadings similar to that of their respective Ni@Pt or Co@Pt counterparts for direct comparison, as can be seen in Table 1.

\subsection{Ethylene Hydrogenation Reactivity}

Figure 1 illustrates the changes in reactivity caused by the overlayer synthesis. The Co@Pt SD sample exhibits a reactivity similar to but less than that of a pure Pt catalyst. The Co@Pt DD and TD samples are both also less reactive than pure Pt, however the trend does not follow the pure Pt, Co@Pt SD trend; the Co@Pt TD sample is more reactive then the Co@Pt DD sample.

\subsection{White Line and EXAFS}

Figures 2 through 4a show X-ray absorption near edge structure (XANES) and extended X-ray absorption fine structure (EXAFS) spectra for all of the cobalt containing catalysts. Figure 2 shows the expanded white line of the $\mathrm{Pt} \mathrm{L}_{\text {III }}$ edge for all Pt containing cobalt samples compared with a $\mathrm{Pt} / \mathrm{Al}_{2} \mathrm{O}_{3}$ reference catalyst. Figure 3 is a comparison of the white line and EXAFS of the Co@Pt DD, bimetallic CoPt and pure Pt. Data for Co@Pt DD is presented in Figure 3 because the low Pt loading of the Co@Pt SD sample resulted in a XANES spectrum with substantial noise. It can be seen in the Pt edge XANES data shown in Figure 2 that, when normalized, the Pt catalyst edge has the lowest white line intensity (initial peak height) and the bimetallic Co-Pt and Co@Pt SD overlayer have the largest peaks. With each additional deposition of $\mathrm{Pt}$ the intensity of the white line is reduced and moves back toward that of the Pt only sample. It can also be seen in both Figures 2 and 3 that the bimetallic Co-Pt sample has a unique feature (shoulder broadening) after the initial white line peak (represented in Figure 2 by " $§$ "). This feature isn't seen in any of the Co@Pt overlayer samples, all of which contain similar Pt loadings. XANES features similar to Figure 2 are seen for the Ni-Pt catalysts (not shown), including the increase in area under the initial peak seen only for the bimetallic catalyst [20]. The Pt-edge EXAFS data for cobalt samples presented in Figure 3 depicts similar wave behavior for the Pt and Co@Pt overlayer sample. Much like with the XANES in Figure 2, the bimetallic Co-Pt sample is dissimilar to both the Pt and 
overlayer sample in that it is out of phase with the other samples. The same trend is seen for the Pt EXAFS in the Ni samples [20].

Figure $4 \mathrm{a}$ shows the Co K-edge XANES spectra for the cobalt containing catalysts. When normalized, the Co-Pt and Co only catalysts have the lowest white line intensity of the Co samples. In contrast to what was seen with the $\mathrm{Pt} \mathrm{L}_{\mathrm{III}}$ edge white line in Figure 2, with each successive overlayer deposition the peak intensity increases. This trend was not seen in the Ni K-edge for the Ni samples; rather the intensity remained similar for all five catalysts (Figure $4 b$ ).

The $\mathrm{k}^{2}$-weighted EXAFS data in k-space for the cobalt samples' Pt LIII-edge and Co K-edge is presented in Figures 5 and 6 . The spectra have been stacked for comparison reasons. Many differences can be seen between the Co parent, Co-Pt bimetallic, and Co@Pt overlayer catalysts and the major ones have been marked on the Figure with numbers 1-5. As was seen in Figure 4a the Co and Co-Pt bimetallic catalysts have data similar to one another. When looking at the Co and Co-Pt bimetallic curves in Figure 6 , the peak labeled 1 is virtually nonexistent and peaks 2 and 4 are both pronounced when compared to the overlayer catalysts. The data above $8 \AA^{-1}$ is also more defined (strong oscillation can be seen) for the Co and Co-Pt when compared to the overlayer catalysts. When looking at the overlayer catalysts it can be seen that with each successive deposition, peaks $2,3,4$, and 5 all become more muted and peak 1 becomes more pronounced. Figure 5 indicates that as the number of Pt depositions increases the amount of $\mathrm{Pt}$ increases on the catalyst. This is seen in the reduced noise and larger oscillations as samples go from Co@Pt SD to Co@Pt TD.

\subsection{Data Fitting using FEFF and Structural Results}

Similarities and differences in the EXAFS function can also be seen in the radial structure plots presented in Figures 7-10. Data for Co samples is presented in Figures 7 and 8, with data for the cobalt catalyst Co K-edge shown in Figure 7 and data for the same catalyst, but $P t L_{111}$ edge in Figure 8 . Figures 9 and 10 present results for the Ni catalysts with the Ni K-edge radial structure plot shown in Figure 9 and the Pt $L_{\| I I}$-edge data of the Ni parent catalysts shown in Figure 10. The primary peak seen in Figure 7 at $\sim 2 \AA$ is the first Co-Co scattering shell and is the peak that was fit using FEFF in order to determine 
structural information about the Co catalysts. Similar data is seen in Figure 9 for the Ni radial structure plot. The radial structure plots, Figures 7-10, all show structural interactions occurring in the overlayer catalysts while the bimetallics, composed of random mixtures of $\mathrm{Ni}-\mathrm{Pt}$ and $\mathrm{Co}-\mathrm{Pt}$, behave similarly to the pure metal $\mathrm{Ni}$ and $\mathrm{Co}$. The resulting values of each fitting variable can be seen in Tables 2-5.

Figures 7 and 9 show the $\mathrm{Co}$ and $\mathrm{Ni} \mathrm{K}$-edge radial structure plots comparing the bimetallic catalyst, the pure parent metal, and a selected overlayer catalyst. The magnitude of the Co-Metal interaction shown in Figure 7 for the overlayer catalysts (Co@Pt SD) is lower than the Co-Co interaction of the pure Co catalyst and the Co-Metal interaction of the bimetallic catalyst. All of the Co@Pt overlayer catalysts $(S D, D D$, and $T D)$ have close to the same magnitude for the Co-Metal interaction. The amount of interaction (coordination number) shown in Table 4 show average coordination numbers of $\sim 6.0$ for the overlayer catalysts which, while lower than the 7.4 of the parent catalyst, are within acceptable error. The lower Parent-Metal interaction seen for the Co overlayer sample is not seen in Figure 9 for the Ni@Pt DD catalyst. In Figure 9, all Ni-Metal interactions at $\sim 2 \AA$ are approximately equivalent, which is reflected in the $\sim 8$ coordination number shown in Table 5.

The $\mathrm{Pt} \mathrm{L}_{\| I}$-edge radial structure data presented in Figure 8 compares a $\mathrm{Pt}$ foil with both the Co-Pt bimetallic and Co@Pt SD overlayer catalysts. Figure 10 shows similar data for the Ni parent catalysts. In Figure 8, The Pt foil curve shows a radial distribution, between $\sim 2.25 \AA$ and $\sim 3.1 \AA$ (not phase corrected). It can be seen that the Co-Pt bimetallic radial plot is different than both the Pt only and overlayer samples in that it has two distinct peaks, one broad peak at $\sim 2.5 \AA$ and another other at $\sim 3.7 \AA$, whereas the Co@Pt sample shows no appreciable peak beyond main peak at $\sim 2.6 \AA$ and the pure Pt spectra shows much sharper peaks than the Co-Pt bimetallic at approximately the same distances $(\sim 2.5 \AA$ and $\sim 3.7 \AA$ respectively, non-phase shifted). Both the bimetallic Co-Pt and Co@Pt overlayer sample show a shift toward shorter radial distances when compared to the Pt foil $(2.58 \AA$, $2.67 \AA$, and $2.76 \AA$ for the Co-Pt, Co@Pt SD, and Pt foil respectively). Similar peak shape and shifts are seen in Figure 10 for the $\mathrm{Ni}$ catalysts. Figure 10 represents the $\mathrm{Pt} \mathrm{L}_{\mathrm{III}}$-edge radial structure plots of $\mathrm{Ni}-\mathrm{Pt}, \mathrm{Ni}$ @ Pt DD, and pure Pt. The same downward shift in radial distances is seen in Figure 10 for the Ni catalysts as is seen in Figure 8 for the Co catalysts. 
The resulting fit plots can be seen in Figures 11-15 and the calculated structural parameters and edge energies can be seen in Tables 2-5. Co-Co and Pt-Metal fits for the Co@Pt SD overlayer catalyst are seen in Figures 11 and 12 respectively. Figure 11 clearly shows that the Co-Co arrangement is a well-defined interaction of one length of Co metallic bond leading to a single isolated peak. Figure 12 , however, displays the Pt-Metal back-transformed Pt $\mathrm{L}_{\mathrm{III}}$-edge for the Co@Pt SD and indicates that the PtMetal interaction is not unique, i.e. the Pt is interacting with neighbors at two or more distances. Backtransformed fits for Ni-Ni and Pt-Metal scattering of the Ni@Pt DD catalyst are also given in Figures 13 and 14. Figure 13 , similarly to Figure 11 and the Co overlayer catalyst, indicates a singular Ni-Metal interaction distance represented by a well-defined isolated Ni-Metal interaction in the radial structure plots (Figure 9). Figure 14 shows the Pt $\mathrm{L}_{\mathrm{III}}$-edge back-transformation of the Ni@Pt DD catalyst and indicates that the Pt overlayer experiences multiple types of Pt-Metal interaction.

\section{4: Discussion}

Figure 1 shows the reactivity of the various catalyst samples for the ethylene hydrogenation descriptor reaction. Previous work has looked at these same catalysts and experimentally determined the apparent activation energy and the reaction order of hydrogen and ethylene in the ethylene hydrogenation descriptor reaction [20]. Skoglund et al. found that as the number of depositions of $\mathrm{Pt}$ increased on the Co@Pt catalysts the reaction order with respect to hydrogen decreased towards the reaction order of hydrogen on a pure $\mathrm{Pt}$ catalyst meaning that surface coverage of hydrogen was increasing. They also showed that, for the same catalysts analyzed here, the apparent activation energy was proportionally related to the reaction order according to hydrogen, i.e. as hydrogen surface coverage increased the reaction order with respect to hydrogen decreased as did the apparent activation energy. Latusek et al., on similar bimetallic overlayer catalysts, showed that the lower the maximum heat of adsorption, the lower the hydrogen TOF [19]. Therefore, the reactivity of the catalysts shown in Figure 1 shows that as the number of $\mathrm{Pt}$ depositions increases the apparent activation energy decreases and the hydrogen coverage increases. As will be shown, the apparent activation energy/hydrogen coverage trends are a result of the Pt $d$-band center $\left(e_{d}\right)$ shift as a result of the overlayer formation of Pt on top of either $\mathrm{Co}$ or Ni particles. The reactivity of the overlayer catalysts (Figure 1) are all similar in TOF as a 
function of temperature to each other and are consistent with the XANES, namely that the $e_{d}$ has shifted downward from the pure Pt leading to lower reactivity. This is most notably seen in the Co@Pt SD reactivity (Figure 1) and XANES peak in Figure 2, respectively. The downward shift in $e_{d}$ of the Pt overlayer, shown as increased white line intensity in Figure 2 (compared to pure Pt), results in a decrease in average energy of the orbitals available for chemical interaction of the Pt atoms with hydrogen. Therefore the reactivity with hydrogen is lower. The bimetallic Co-Pt, as shown in Figure 1 , has a reactivity similar to what could be conceptualized as the linear combination of the pure Pt and pure Co catalysts. However, considering that the bimetallic has a Pt loading in line with the overlayer samples, the lower reactivity is most likely solely a result of the Pt on the surface as opposed to being a result of some interaction between the Co and Pt. The Co@Pt SD sample has a lower reactivity then the pure Pt catalyst and the Co@Pt DD and TD have even lower reactivity. However, the TD sample is slightly out of trend with the SD and DD samples, possibly because as the synthesis procedure was repeated a third time, individual Pt crystallites were formed and as a result the reactivity of the catalyst was increased. The formation of Pt crystallites should result in activity similar to pure Pt. Although this is not shown in Figure 1, the TOF in the figure were calculated using the total amount of Pt present (from elemental analysis). Since the XAS evidence indicates Pt crystallite formation, determining TOF based on total Pt present assumes all Pt is on the surface, which is unlikely for the Co@Pt TD sample. Thus, the active Pt is overcounted and the true TOF is probably closer to that of pure Pt. This reactivity trend is consistent with the white line intensity trend found in Figure 2, possibly corroborating the Pt $e_{d}$ shift seen in the XANES data and confirming the synthesis technique as capable of creating Pt overlayers atop of $\mathrm{Ni}$ and $\mathrm{Co}$.

White line peak intensity in XAS experiments can be correlated with d-band vacancies in the emitting atom [35-39]. This characteristic has been shown specifically for the $\mathrm{Pt} \mathrm{L}_{\mathrm{III}}$-edge, in that with an increase in Pt d-band vacancies, white line peak intensity will increase [39]; this also holds true for many other transition metals such as palladium, cobalt, and nickel [24, 40-42]. However, in the Co@Pt system the calculated $d$-band vacancy has been shown to be less important than the charge transfer from Co to $\mathrm{Pt}$ as a result of the higher electronegativity of $\mathrm{Pt}$ [43]. While $\mathrm{Ni}$ and $\mathrm{Co}$ share similar electronegativities, Co has a slightly lower electronegativity compared to $\mathrm{Ni}$ and will therefore be more effected by the electron withdrawing capability of Pt. In the Co@Pt and Ni@Pt systems it has been shown that the d- 
band center $\left(e_{d}\right)$ is the more important indicator of reactivity [43]. The Pt deposited on Co samples shown in Figure 2, show how the white line changes with $e_{d}$ change. The more Pt overlayed on the Co i.e., the double and triple deposition samples, the more Pt like the sample becomes. The single deposition Co@Pt sample appears to best represent this $e_{d}$ shift and the compressive strain of overlaying a larger atom (Pt: $2.77 \AA$ ) on top of one with a smaller interatomic distance (Co: $2.49 \AA$ ) as Greeley et al. noted [44]. Again, with the double and triple deposition samples the samples become more Pt like; there is less compressive strain exerted on the Pt overlayer by the Co parent metal due to the addition of more Pt, i.e. the Pt has more bulk-like characteristics due to the existence of multiple Pt layers, and the white line is similar to the white line of the pure Pt catalyst, meaning that with more Pt depositions small Pt crystallites are formed. Concerning the bimetallic samples, the increase in area under the XANES peak shown in Figure 3 is consistent with literature for bimetallic Co-Pt and Ni-Pt catalysts, and is especially pronounced at lower Pt loadings [40, 41, 45]. Figure 3 also shows the pure Pt catalysts and the Co@Pt DD catalysts have a similar structure to each other as indicated by wave structure and they are both dissimilar to the Co-Pt bimetallic which is out of phase. This indicates that the Pt in both the pure Pt catalysts and the Co@Pt DD overlayer catalyst is in a similar physical environment, and more importantly that the Pt in Co@Pt DD is in a pure Pt environment. This, paired with the reactivity data shown in Figure 1, once again indicates that bimetallic overlayer samples have been synthesized.

With the downward shift in the $e_{d}$ of the Pt there is a reciprocal upward shift in the $d$-band center of the Co [46], this is seen in the increasing white line intensities in Figure 4a and in the increasing edge energies shown in Table 4. Figure 4b shows the same data, but for the Ni catalysts (Inset in both Figures $4 \mathrm{a}$ and $4 \mathrm{~b}$ is the XANES spectra to show that no pre-edge oxide features exist and that the parent metals, $\mathrm{Ni}$ and Co, are fully reduced. Table 4 shows the Co-Pt, Co@Pt SD, Co@Pt DD, and Co@Pt TD as having an edge approximately $3 \mathrm{eV}$ greater than Co foil. A cobalt oxide (CoO) standard was analyzed and found to have an edge energy of $7722.3 \mathrm{eV}, \sim 10 \mathrm{eV}$ greater than Co foil, indicating that the samples were fully reduced, similar to work done by Skoglund et al. [20]). In contrast with the Co samples, there is no trend in white line intensity (Figure 4a). This may be a result from $\mathrm{Ni}$ having the same number of electrons in its valence shell as Pt while Co has one less. Therefore, Co has one more bonding molecular orbital that is open and lower in energy, as compared to $\mathrm{Ni}$, causing the d-band width to increase even more for the 
Co@Pt samples and therefore the Pt $e_{d}$ shifts downwards farther than it does in the Ni@Pt system. This phenomenon is consistent with calculations conducted by Ruban et al. [47] and by the edge energies shown in Tables 4 and 5. In addition, the changes in white line height shown in Figures $4 \mathrm{a}$ and $4 \mathrm{~b}$ are consistent with the changes in edge energy, or lack thereof in the case of the Ni parent metal, shown in Tables 4 and 5. The bimetallic Co-Pt and the pure Co catalyst have similar white line intensity as a result of the lack of Pt interaction causing the Co $e_{d}$ to shift upwards. The Co in the overlayer samples are interacting with the Pt and the upshift in the $\mathrm{Co} \mathrm{e}_{\mathrm{d}}$ is apparent in Figure 4a as the Co K-edge white line intensity increases with the number of Pt depositions. Both Figures 2 and $4 a$ show that the overlayer samples are definitely not just bimetallic mixtures, but rather behave similar to overlayer systems where the two metals are in intimate contact with one another and are experiencing some synergistic effects. The changes in white line intensity at both the Pt LIII-edge and the $\mathrm{Ni}$ and Co K-edges are shown as a function of the number of depositions in Figures 2, 4a, and $4 \mathrm{~b}$ respectively. Table 1 shows that even as the number of $\mathrm{Pt}$ depositions increase on the identical loaded respective parent metal catalyst, the metal dispersion, and therefore the approximate particle size, stays consistent throughout, indicating that the quantum size effect is not the phenomenon represented by the changes in white line or edge energy for any of the three metals analyzed. The changes in white line and edge energy between the parent metal and overlayer Pt seen in the XAS results is predominantly the result of the electronic interaction between the parent and overlayer metals.

The decrease in Pt white line intensity with overlayer depositions is also consistent with computational work which predicts that an overlayer of $\mathrm{Pt}$ atop a $\mathrm{Ni}$ or Co base metal will result in a negative shift in the center of the Pt d-band and ultimately result in more Pt d-band vacancies [18]. This increase in Pt d-band vacancies can also be correlated with a reduction in adsorbate binding strength (namely, $\mathrm{H}_{2}$ and $\mathrm{CO}$ ) as has been demonstrated for the same overlayer catalysts as examined in this paper [20].

The Pt d-band shift caused by the presence of $\mathrm{Co}$ or Ni corresponding with to shorter Pt-Metal bond distances has also been seen in other XAS studies for bimetallic catalysts, which are especially pronounced when Pt loadings are low $[6,40,41]$. Due to the Co-Pt and Ni-Pt bimetallic samples' unique radial structure peaks, fitting the samples to a Pt FEFF model proved difficult. It was assumed that the Pt 
atoms were highly disperse throughout the bimetallic catalysts and most likely not in large Pt clusters. In order to fit the bimetallic catalysts, a model was created which consisted of a single Pt scattering atom in the center of a Co or Ni crystal keeping the interatomic distances fixed for Co or Ni. While this model provided a better fit than that of a Pt crystal, the fit still resulted in non-ideal fit statistics (i.e. a large Rfactor meaning a large error in the fit relative to the empirical data) indicating that this model is not strongly descriptive of our sample. Two, three, four and five atom Pt crystallites in Ni and Co crystals were also attempted but the fit statistics did not improve indicating that the bimetallics contain very disperse Pt atoms. All of the fitting regarding both the Ni-Pt and Co-Pt bimetallic systems indicated Pt needed to be be relaxed inside the crystal it was placed. In the future, a possible solution to this problem are simple molecular dynamics calculations allowing for the relaxation of the crystal in the Ni-Pt and Co-Pt systems. The computationally relaxed structures, used as FEFF models, could improve fit statistics for the bimetallic systems.

The lack of high wave number oscillation in the Co@Pt DD and TD samples compared to the SD and parent $\mathrm{CO}$ in Figure 6 is unclear. It may be the result of more Pt interaction with the Co interrupting the long range structure of Co. The lack of oscillation in the EXAFS region for the Co@Pt DD and TD samples does not appear to result from inconsistent data normalization or fitting procedures, rather it is indicative of the physical structure of the samples. The clear differences seen in Figure 6 between the Co parent catalyst and all the Co@Pt overlayer catalysts could be the result of Pt being deposited atop the Co in an overlayer formation, ultimately affecting the EXAFS of the Co K-edge. Overlayer formation is also evident in the Pt radial structure plots (Figures 8 and 10) along with calculated coordination numbers and radial distances presented in Tables 2 and 3. The $P t L_{11}$-edge radial structure plots shown in Figures 8 and 10 for $\mathrm{Co}$ and $\mathrm{Ni}$ samples, respectively, show a negative shift in radial distance for the main peak of both the bimetallic and overlayer catalysts when compared to the Pt only catalyst. This decrease in radial distance for the first Pt scattering shell is an expected result of combining Pt with another metal with shorter lattice distances, in this case $\mathrm{Ni}$ or $\mathrm{Co}$, and is documented in literature [6, 40, 42, 48]. This decrease in Pt-Metal bond distance is especially pronounced at low Pt loadings $[40,48]$, as the Pt is unable to create large Pt ensembles. Radial structure data shown in Figures 8 and 10 also gives insight into the atomic neighborhood of the Pt in each catalyst. The bimetallic Co-Pt and Ni-Pt samples display a 
strong first Pt scattering shell peak at $\sim 2.25 \AA$. This peak represents Pt most likely in a slightly distorted Co or Ni matrix. This is also consistent with the fitting parameters in Tables 1 and 2 which show Pt-Metal distances of $2.54 \AA$ and $2.58 \AA$ that are intermediate to Pt-Pt $(2.77 \AA)$ and Co-Co $(2.49 \AA)$ or Ni-Ni $(2.50$ $\AA$ A). The Pt only catalyst shows a nearest neighbor peak at $\sim 2.5-3.25 \AA$ consistent with the expected Pt nearest neighbor distance of $2.77 \AA$. Finally, the overlayer catalysts show a broader peak from $\sim 2-3 \AA$ for the nearest neighbor interaction. This could indicate that the bimetallic Co-Pt and $\mathrm{Ni}-\mathrm{Pt}$ samples have small highly disperse $\mathrm{Pt}$ atoms causing the scattering behavior to be confined to nearly all Co or $\mathrm{Ni}$ neighbors. Whereas, the overlayers and Pt only samples consist of Pt clusters allowing for scattering with a combination of Pt neighbors at larger distances and Co or Ni neighbors at shorter distances. Larger Pt clusters in overlayer catalyst at similar loadings to their bimetallic counterparts could indicate that overlayer depositions have only allowed the Pt to deposit atop the Ni or Co metal resulting in less overall Pt dispersion and larger Pt crystallite sizes.

Values consistent with the shift in radial structure plots are seen in calculated fitting parameters given Tables 2 and 3. The Pt only catalyst has a longer Pt-Pt bond distance than most of the other catalyst samples and compares well with the bulk Pt-Pt bond distance of $2.77 \AA$ and literature values for similar catalysts [49-51]. With each successive overlayer deposition on both the $\mathrm{Ni}$ and Co parent calalysts the Pt-M bond distance typically increased. For the Co overlayer catalyst samples the Pt-Metal bond distance started at $\sim 2.67 \AA$ for the Co@Pt SD sample as compared to Pt-Metal bond distance of 2.79 for the Co@Pt TD sample. The bond distance of the Co@Pt SD sample (2.67 $⿱$ $)$ is only slightly larger $(0.01 \AA)$ than the combination of the atomic radii of metallic Co and Pt, $2.66 \AA$. The increase in bond distance with Pt depositions could be due to the addition of more Pt to the catalyst allowing for the deposition of $\mathrm{Pt}$ on the existing $\mathrm{Pt}$ instead of on the parent metal as desired. This allows formation of larger Pt crystals atop the parent metal, allowing for Pt-Pt bond distances to relax back toward that of Pt alone. Larger Pt ensembles can also be confirmed by the coordination number values $(\mathrm{N})$ shown in Tables 2 and 3. The coordination number started at 5.6 and 6.3, respectively, for the Co@Pt SD and $\mathrm{Ni@Pt} \mathrm{SD} \mathrm{catalysts.} \mathrm{These} \mathrm{values} \mathrm{are} \mathrm{less} \mathrm{than} \mathrm{the} 6.1$ and $8.2 \mathrm{CN}$ for the parent Co and $\mathrm{Ni}$ catalysts. Since the overlayers were deposited on these parent catalysts, the lower CN provides some evidence that the $\mathrm{Pt}$ is surface segregated. With each successive deposition overlayer $\mathrm{CN}$ values increase 
indicating an increase in Pt neighbors and ultimately larger Pt crystallites. For the Co overlayer catalyst the coordination number went from 5.6 to 7.7 for the Co@Pt SD and Co@Pt TD samples respectively. For the Ni overlayer catalyst the coordination number went from 6.3 to 8.2 for the Ni@Pt SD and Ni@Pt TD samples respectively. The increasing $\mathrm{CN}$ and the interatomic distance as overlayer Pt deposition is repeated together indicates the formation of Pt crystallites.

The Co and Ni K-edge radial structure plots are given in Figures 7 and 9 respectively and illustrate a different aspect of the overlayer catalyst system. Unlike what was seen in the Pt $\mathrm{L}_{\| I I}$-edge radial structure plot, the radial structure plots for the parent metals remain similar for all of the samples. This is to be expected as there is substantially more parent metal (Co or Ni) deposited on the catalyst than there is $\mathrm{Pt}$. This allows for large crystals of the parent metal, causing the majority of the Co or $\mathrm{Ni}$ atoms to be surrounded by like atoms and ultimately not affected by deposition of Pt. Similar trends are evident from the fitting values presented in Tables 4 and 5 which show Co-Co and Ni-Ni bond distance and coordination number. Tables 4 and 5 concern the parent metals themselves and are consistent with the fact that the same parent metal sample for both $\mathrm{Ni}$ and $\mathrm{Co}$ were used as the starting place for the synthesis of the overlayer samples. While the interatomic distances of $\mathrm{Ni}$ and $\mathrm{Co}$ shown in Tables 4 and 5 are consistent for each metal, and the trend across the two metals is corroborated by literature, the Co is slightly longer than literature would suggest [52]. The long bond distances calculated for Co and shown in Table 4 might be indicative of alloying. However, the formation of Co-Pt alloy seems unlikely due to the lower surface energy of Pt. Bardi et al. have shown that even in samples synthesized to be Co-Pt alloys, the $\mathrm{Pt}$ in the first layer or two of the crystalline structure migrates to the surface creating a surface of pure $\mathrm{Pt}$ and a region immediately sub-surface of pure Co [53]. The low concentration of $\mathrm{Pt}$ is also a reason the Pt would be unlikely to alloy with the Co. The similarly prepared Ni catalysts also show no evidence of alloying. The $\mathrm{Ni}$ catalysts has a higher coordination number than the Co catalysts and therefore has a metallic radii closer to that of $\mathrm{Ni}$ foil. Evidence of the parent metal being highly reduced and not in a metal-oxide is also evident by the radial structure plots presented in Figures 7 and 9 . When compared to literature, the Co and $\mathrm{Ni}$ radial structure plots align with that of reduced cobalt and not with that of any cobalt or nickel oxide species, indicating that reduction at $400^{\circ} \mathrm{C}$ has been sufficient $[41,54]$. 
All of the structural and electronic characteristics of the catalysts examined with XAS and discussed above relate back to the ethylene hydrogenation reactivity experiments conducted on the pure Pt, Co-Pt bimetallic and Co@Pt overlayer catalysts. As the Pt d-band is affected by the parent metal the reactivity of the catalyst is decreased. And, as shown in Figure 2, as more Pt is deposited atop the parent metal the catalyst starts to exhibit electronic behavior more like bulk $\mathrm{Pt}$, which can be seen in Figure 1 in how the reactivity of the Co@Pt TD catalyst moves towards the reactivity characteristics of pure Pt as compared to Co@Pt DD.

\section{5: Conclusions}

XAS studies have been conducted in order to further characterize Co@Pt and Ni@Pt overlayer catalysts. Through the use of XANES and EXAFS analysis with FEFF fitting and the use of the ethylene hydrogenation descriptor reaction we have been able to show that the directed deposition overlayer synthesis technique is capable of depositing Pt atop a parent metal such as Co or Ni and does not merely create a bimetallic catalyst. The SD catalysts were found to exhibit a reactivity lower than that of pure Pt, higher than pure parent metal and dissimilar to that of the bimetallic catalysts ( $\mathrm{Ni}-\mathrm{Pt}$ and Co-Pt). Additionally the XANES corroborated the reactivity data, showing the SD catalysts had the largest d-band shift as compared to the DD and TD catalysts. Multiple overlayer depositions led to changes in the Pt $L_{\text {III- }}$ edge consistent with computational predictions and allow for further tailoring of overlayer systems. With successive depositions, white line intensity decreased toward that of $\mathrm{Pt}$ only, coordination number increased, and Pt-Pt bond length increased. This could be evidence that with successive depositions larger Pt clusters, moving toward bulk characteristics, are formed although overlayer characteristics remain.

\section{Acknowledgements}

The authors would like to acknowledge funding support from Funding provided by National Science Foundation, Chemical, Bioengineering, Environmental and Transport Systems [CBET-0933017]. 
MRCAT operations are supported by the Department of Energy and the MRCAT member institutions. Dr. Jeff Miller for all of the XAS sampling equipment.

Use of the Advanced Photon Source, an Office of Science User Facility operated for the U.S. Department of Energy (DOE) Office of Science by Argonne National Laboratory, was supported by the U.S. DOE under Contract No. DE-AC02-06CH11357.

This material is based upon work supported by the University of Wyoming School of Energy Resources through its Graduate Assistantship program. 


\section{References:}

1. Navarro RM, Pawelec B, Trejo JM, Mariscal R, Fierro JLG, J. Catal. 189 (2000) 184.

2. Prüsse U, Vorlop K-D, J. Mol. Catal. A 173 (2001) 313.

3. Coq B, Figueras F, J. Mol. Catal. A 173 (2001) 117.

4. Carter JL, McVicker GB, Weissman W, Kmak WS, Sinfelt JH, Appl. Catal. 3 (1982) 327.

5. Burch R, Mitchell AJ, Appl. Catal. 6 (1983) 121.

6. Massard R, Uzio D, Thomazeau C, Pichon C, Rousset JL, Bertolini JC, J. Catal. 245 (2007)133.

7. Juszczyk W, Karpinski Z, Appl. Catal. A 206 (2001) 67.

8. Chen JG, Menning CA, Zellner MB, Surf. Sci. Rep. 63 (2008) 201.

9. Skoplyak O, Barteau MA, Chen JG, J. Phys. Chem. B 110 (2006) 1686.

10. Kitchin JR, Khan NA, Barteau MA, Chen JG, Yakshinskiy B, Madey TE, Surf. Sci. 544 (2003) 295.

11. Hwu HH, Eng J, Chen JG, J. Am. Chem. Soc. 124 (2002) 702.

12. Khan NA, Hwu HH, Chen JG, J. Catal. 205 (2002) 259.

13. Pallassana V, Neurock M, Coulston GW, J. Phys. Chem. B 103 (1999) 8973.

14. Pallassana V, Neurock M, J. Catal. 191 (2000) 301.

15. Pallassana V, Neurock M, Hansen LB, Hammer B, Norskov JK, Phys. Rev. B 60 (1999) 6146.

16. Zellner MB, Chen JG, J. Catal. 235(2005) 393.

17. Zellner MB, Goda AM, Skoplyak O, Barteau MA, Chen JG, Surf. Sci. 583 (2005) 281.

18. Christoffersen E, Liu P, Ruban A, Skriver HL, Norskov JK, J. Catal. 199 (2001) 123.

19. Latusek MP, Spigarelli BP, Heimerl RM, Holles JH, J. Catal. 263 (2009) 306.

20. Skoglund MD, Jackson CL, McKim KJ, Olsen HJ, Sabirzyanov S, Holles JH, Appl. Catal. A 46(2013) 355.

21. Skoglund MD, Holles JH, Catal. Lett. 143(2013) 966.

22. Womes M, Cholley T, Le Peltier F, Morin S, Didillon B, Szydlowski-Schildknecht N, Appl. Catal. A 283 (2005) 9.

23. Bazin D, Guczi L, Recent Res. Dev. Phys. Chem. 3 (1999) 387. 
24. Holles JH, Davis RJ, J. Phys. Chem. B 104 (2000) 9653.

25. Shibata T, Bunker BA, Zhang Z, Meisel D, Vardeman II CF, Gezelter JD, J. Am. Chem. Soc. 124 (2002) 11989.

26. Vijay S, Wolf EE, Miller JT, Kropf AJ, Appl. Catal. A 264 (2004) 125.

27. Van Zon JBAD, Koningsberger DC, van't Blik HFJ, Sayers DE, J. Chem. Phys. 82 (1985) 5742.

28. Cook JW Jr, Sayers DE, J. Appl. Phys. 52 (1981) 5024.

29. Ravel B, Newville M, J. Syncrotron. Radiat. 12 (2005) 537.

30. Downs RT, Hall-Wallace M, Am. Mineral 88 (2003) 247.

31. Suh I-K, Ohta H, Waseda Y, J. Mater. Sci. 23 (1988) 757.

32. Wyckoff RWG (1963) Crystal Structures, Second Ed. Interscience Publishers, New York, New York

33. Davey WP, Phys. Rev. 25 (1925) 753.

34. Koningsberger DC, Mojet BL, van Dorssen GE, Ramaker DE, Top. Catal. 10 (2000) 143.

35. Mansour AN, Cook JW, Sayers DE, J. Phys. Chem. 88 (1984) 2330.

36. Blum L, Abruna HD, J. W, Gordon II JG, Borges GL, Samant MG, Melroy OR, J. Chem. Phys. 85 (1986) 6732

37. McBreen J, O'Grady WE, Tourillon G, Dartyge E, Fontaine A, J. Electroanal. Chem. 307 (1991) 229.

38. Melroy OR, Samant MG, Borges GL, Gordon II JG, Blum L, White JH, Albarelli MJ, McMillan M, Abruna HD, Langmuir 4 (1988) 728.

39. Mukerjee S, Srinivasan S, Soriaga MP, McBreen J, J. Electrochem. Soc. 142 (1995) 1409.

40. Bommannavar AS, Montano PA, Yacaman MJ, Surf. Sci .156 (1985) 426.

41. Jacobs G, Chaney JA, Patterson PM, Das TK, Maillot JC, Davis BH, .J Synchrotron Rad. 11 (2004) 414.

42. Cheney BA, Lauterbach JA, Chen JG, Appl. Catal. A 394 (2011) 41.

43. Wieckowski A (2009) Fuel Cell Catalysis: A Surface Science Approach. Wiley, Hoboken, N.J

44. Greeley J, Norskov JK, Mavrikakis M, Annu. Rev. Phys. Chem. 53 (2002) 319.

45. Guczi L, Bazin D, Kovacs I, et al, Top. Catal. 20 (2002) 129.

46. Mukerjee S, Srinivasan S, Soriaga MP, McBreen J, J. Electrochem. Soc. 142 (1995) 1409.

47. Ruban A, Hammer B, Stoltze P, Skriver HL, Norskov JK, J. Mol. Catal. Chem. 115 (1997) 421. 
48. Huang Q, Yang H, Tang Y, Lu T, Akins DL, Electrochem. Commun. 8 (2006) 1220.

49. Ramallo-López JM, Santori GF, Giovanetti L, Casella ML, Ferretti OA, Requejo FG, J Phys. Chem. B 107 (2003) 11441.

50. Guyot-Sionnest NS, Villain F, Bazin D, Dexpert H, Catal. Lett. 8 (1991) 283.

51. Borgna A, Le Normand F, Garetto T, Apesteguia CR, Moraweck B, Catal. Lett. 1 (1992) 175.

52. Greenwood NN, Earnshaw A (1997) Chemistry of the Elements, 2nd Edition. ButterworthHeinemann

53. Bardi U, Beard BC, Ross PN, J Catal. 124 (1990) 22.

54. McBreen J, O'Grady WE, Pandya KI, Hoffman RW, Sayers DE, Langmuir 3 (1987) 428. 


\section{Figure Captions:}

Figure 1: Alumina supported cobalt catalyst Arrhenius-type plot for ethylene hydrogenation

Figure 2: Enlargements of Normalized Pt $\mathrm{L}_{111}$ edge white line plots for Pt, Co@Pt overlayers, and bimetallic Co-Pt catalysts. Plots are offset in energy for clarity. Unique bimetallic feature labeled with "§".

Figure 3: Normalized Pt $\mathrm{L}_{\| 1}$-edge EXAFS energy plots for Pt, Co@Pt, and bimetallic Co-Pt catalysts.

Figure 4a: Enlargements of Normalized Co K-edge white line plots for Co, Co@Pt overlayers, and bimetallic Co-Pt catalysts. Inset: XANES spectra for the same catalysts. Plots are offset in energy for clarity.

Figure 4b: Enlargements of Normalized Ni K-edge white line plots for $\mathrm{Ni}$, Ni@ Pt overlayers, and bimetallic Ni-Pt catalysts. Inset: XANES spectra for the same catalysts. Plots are offset in energy for clarity.

Figure 5: Unfiltered $\mathrm{Pt} \mathrm{L}_{\text {III }} \mathrm{Edge} \mathrm{X}\left(\mathrm{k}^{2}\right)$ data for all cobalt containing catalysts.

Figure 6: Unfiltered Co K-Edge $\mathrm{X}\left(\mathrm{k}^{2}\right)$ data for all cobalt catalysts.

Figure 7: Radial structure function (not corrected for phase shifts) derived from the Fourier transforms of $X k^{2}$-weighted EXAFS of the Co K-edge for Co, Co-Pt bimetallic, and Co@Pt SD overlayer catalysts. Individual peaks are not labeled, the labels indicate the entire dataset.

Figure 8: Radial structure function (not corrected for phase shifts) derived from the Fourier transforms of $X k^{2}$-weighted EXAFS of the Pt $\mathrm{L}_{11}$-edge for Co, Co-Pt bimetallic, and Co@Pt SD overlayer catalysts. Second Pt-Pt scattering shell peak labeled with "ه". Individual peaks are not labeled, the labels indicate the entire dataset.

Figure 9: Radial structure function (not corrected for phase shifts) derived from the Fourier transforms of $X k^{2}$-weighted EXAFS of the Ni K-edge for Ni, Ni-Pt bimetallic, and Ni@Pt DD overlayer catalysts. Individual peaks are not labeled, the labels indicate the entire dataset.

Figure 10: Radial structure function (not corrected for phase shifts) derived from the Fourier transforms of $X k^{2}$-weighted EXAFS of the Pt $\mathrm{L}_{\mathrm{III}}$-edge for $\mathrm{Ni}$, Ni-Pt bimetallic, and Ni@Pt DD overlayer catalysts. Individual peaks are not labeled, the labels indicate the entire dataset.

Figure 11: Fourier-filtered EXAFS function of the Co-Co shell and the FEFF first scattering shell curve fit used to calculate structural parameters for Co@Pt SD catalyst.

Figure 12: Fourier-filtered EXAFS function of the Pt-Pt shell and the FEFF first and second scattering shell curve fit used to calculate structural parameters for Co@Pt SD catalyst.

Figure 13: Fourier-filtered EXAFS function of the Ni-Ni shell and the FEFF first scattering shell curve fit used to calculate structural parameters for $\mathrm{Ni@Pt} \mathrm{DD} \mathrm{catalyst.}$

Figure 14: Fourier-filtered EXAFS function of the Pt-Pt shell and the FEFF first and second scattering shell curve fit used to calculate structural parameters for Ni@Pt DD catalyst. 


\section{Table Captions:}

Table 1: Metal loading of catalyst samples.

Table 2: Platinum EXAFS results for Pt-Pt in Pt, Co-Pt bimetallic, and Co@Pt overlayer catalysts.

Table 3: Platinum EXAFS results for Pt-Pt in Pt, Ni-Pt bimetallic, and Ni@Pt overlayer catalysts.

Table 4: Cobalt EXAFS results for Co-Co in Pt, Co-Pt bimetallic, and Co@ Pt overlayer catalysts.

Table 5: Nickel EXAFS results for Ni-Ni in Pt, Ni-Pt bimetallic, and Ni@Pt overlayer catalysts. 
Figure 1:

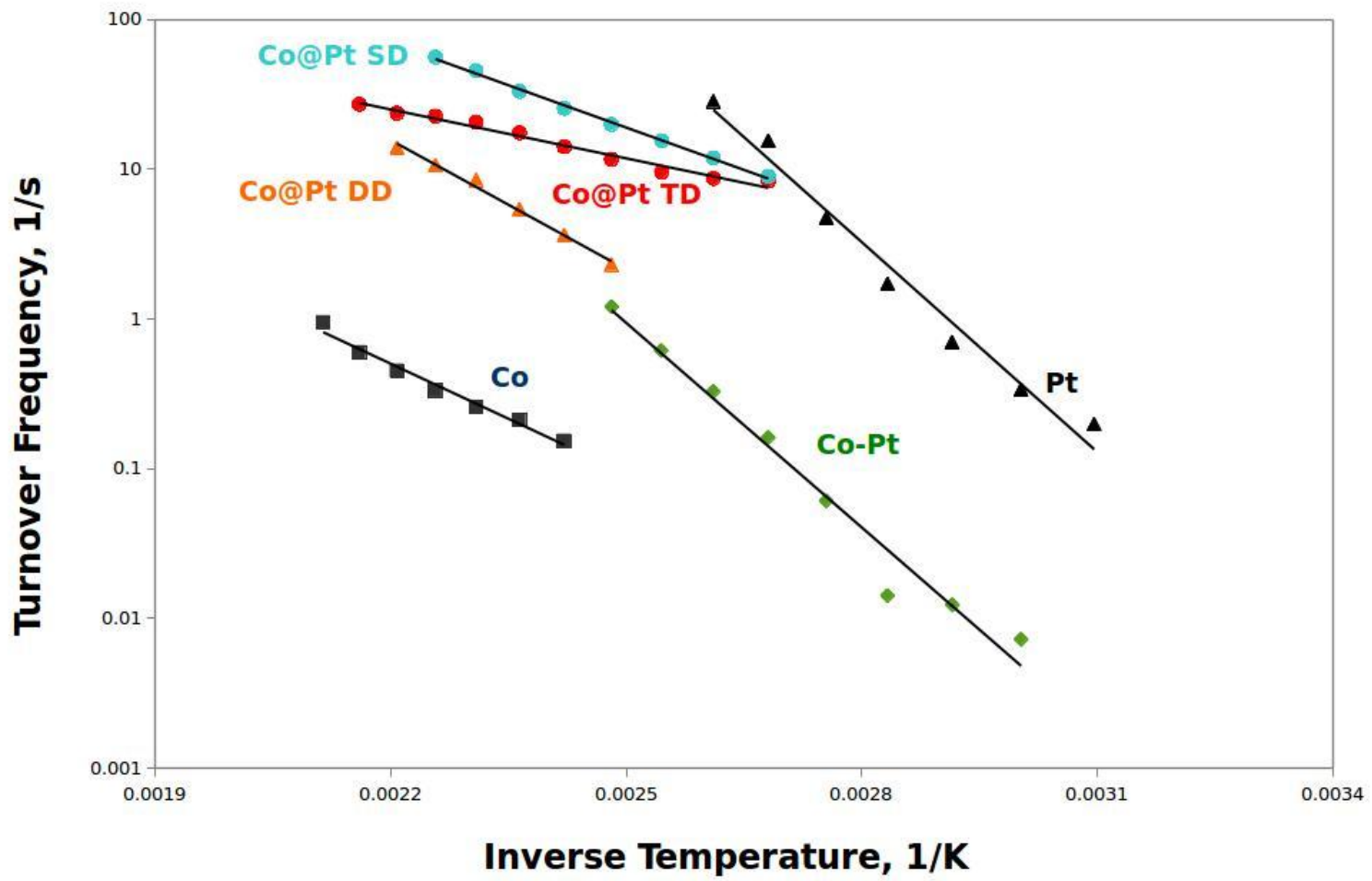


Figure 2:

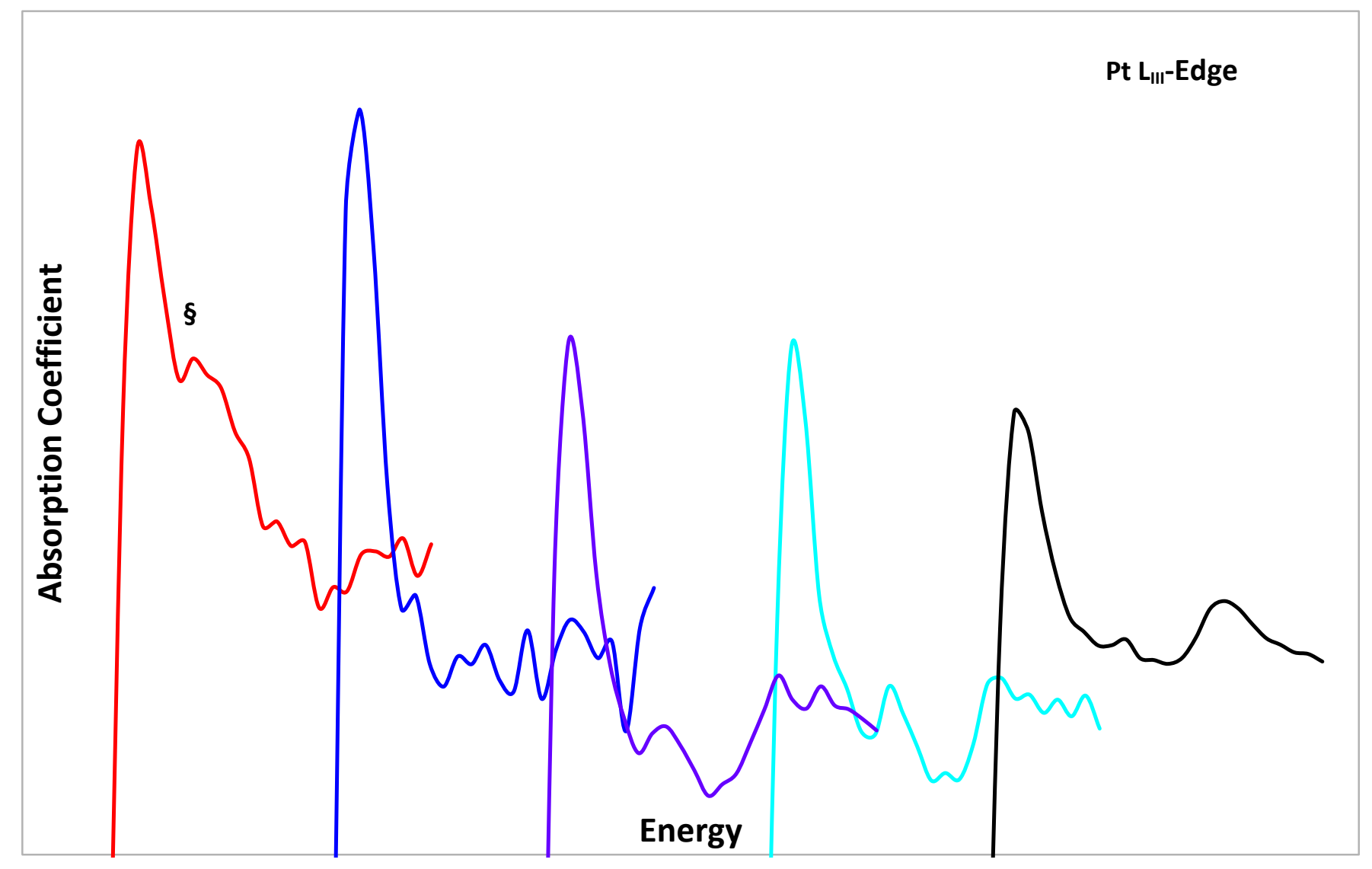


Figure 3:

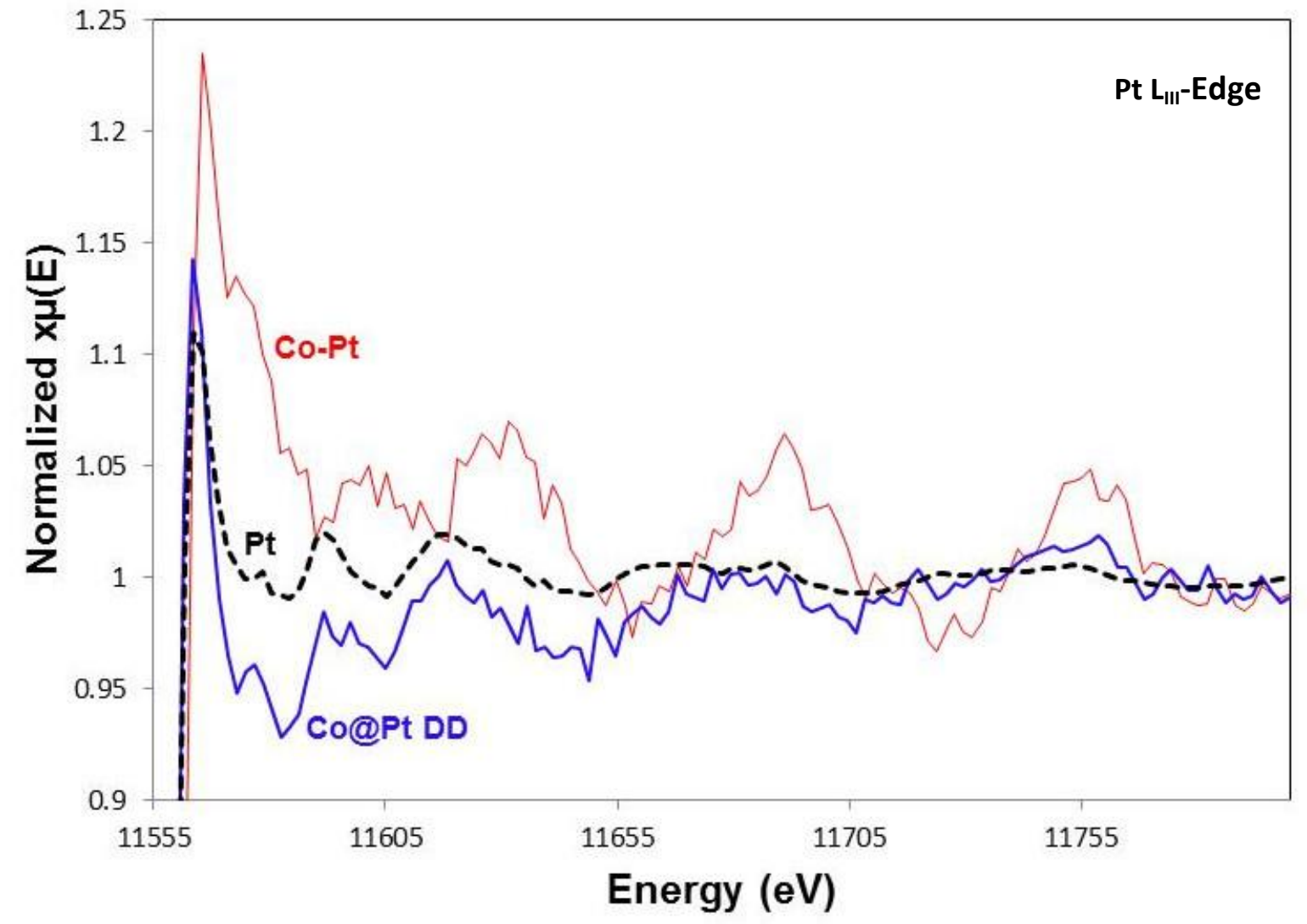


Figure 4a:

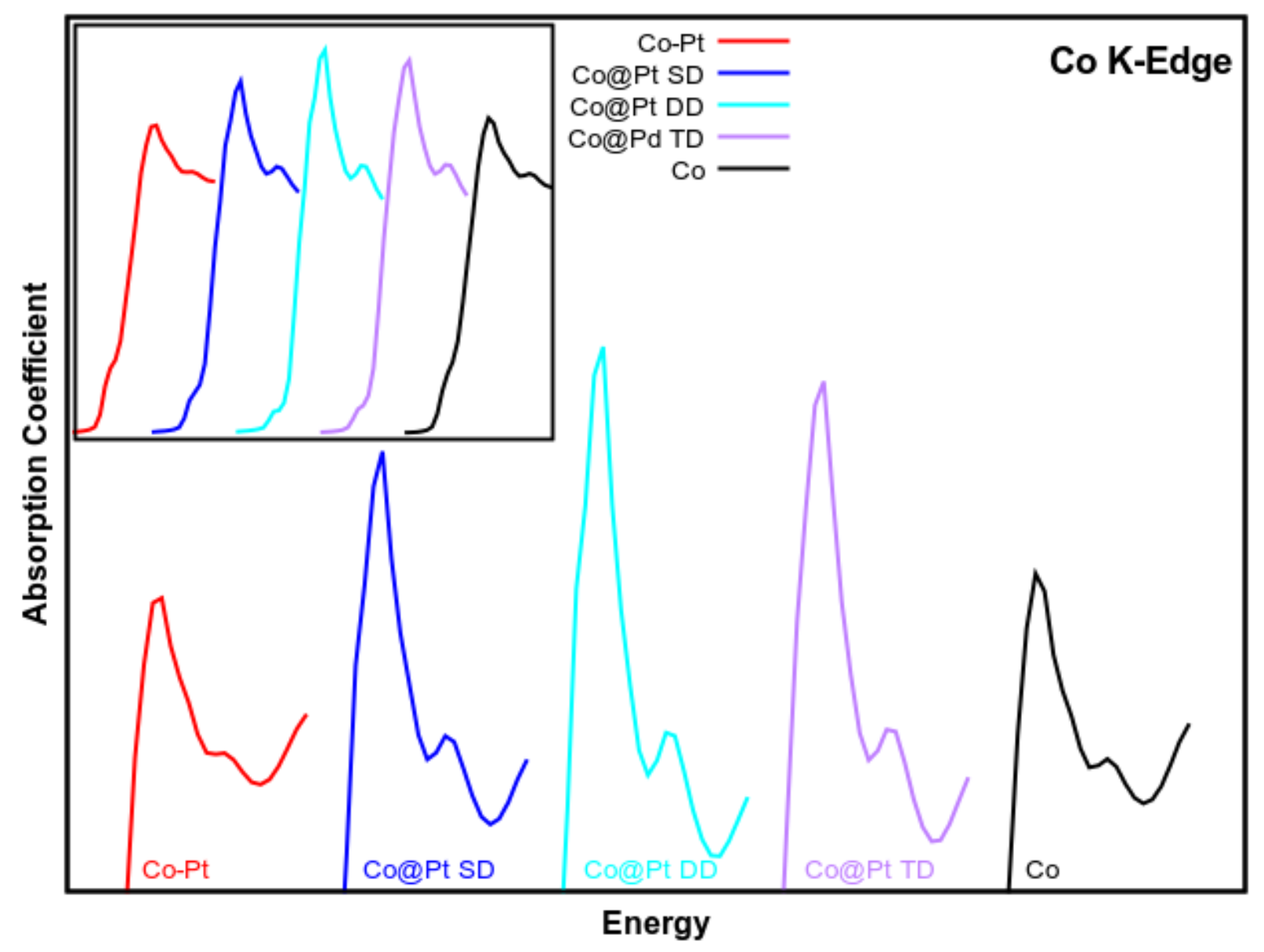


Figure 4b:

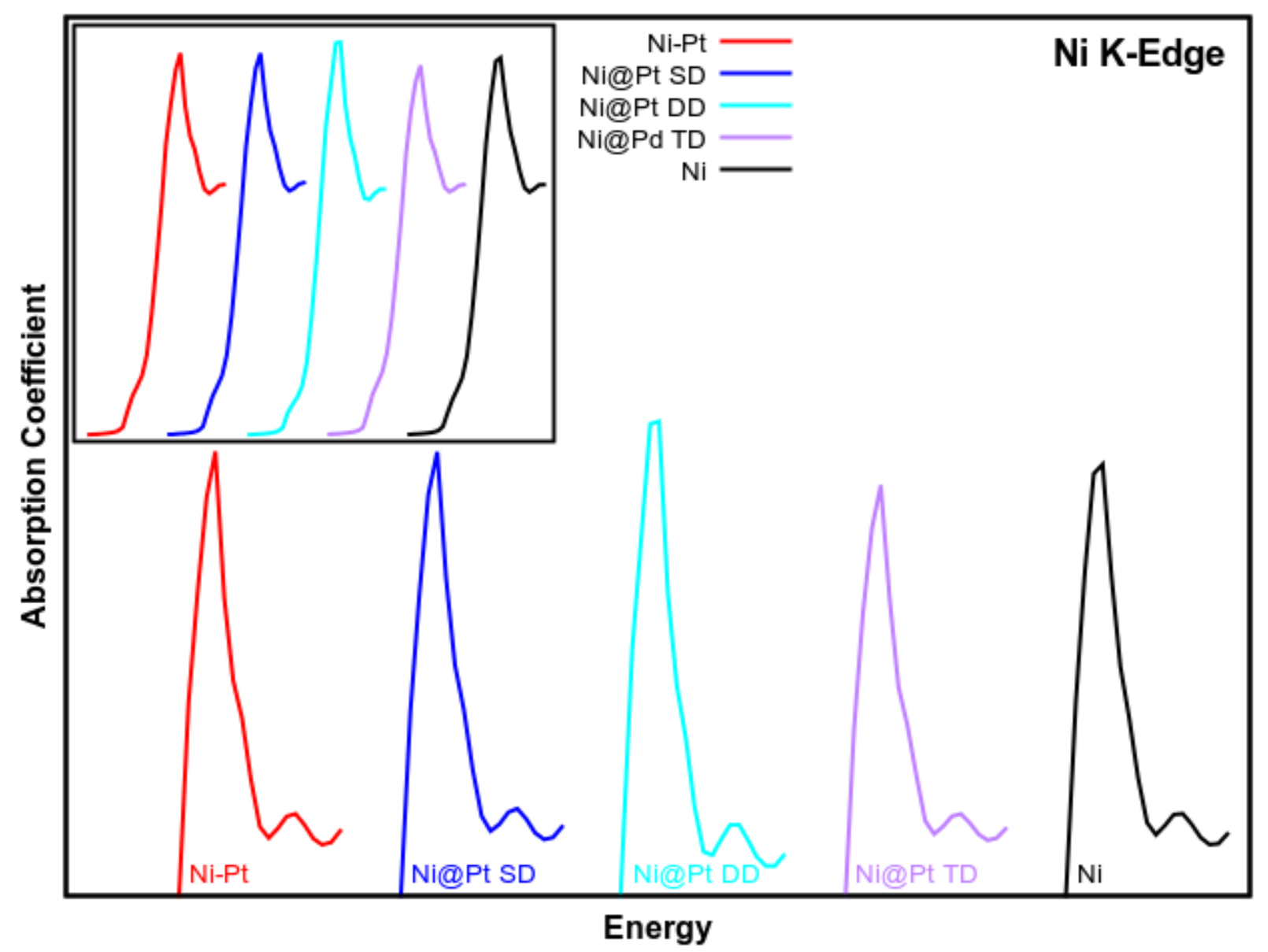


Figure 5:

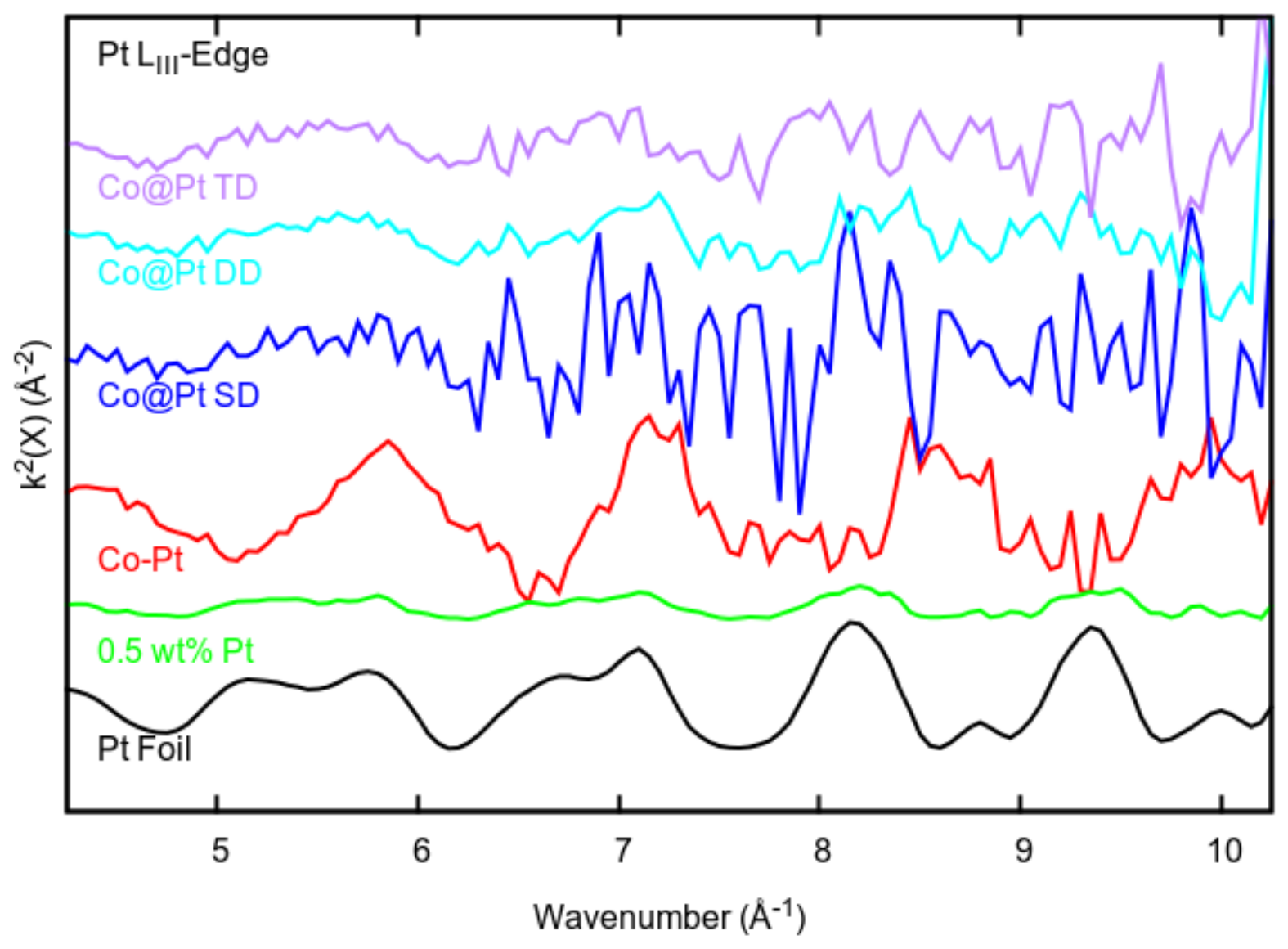




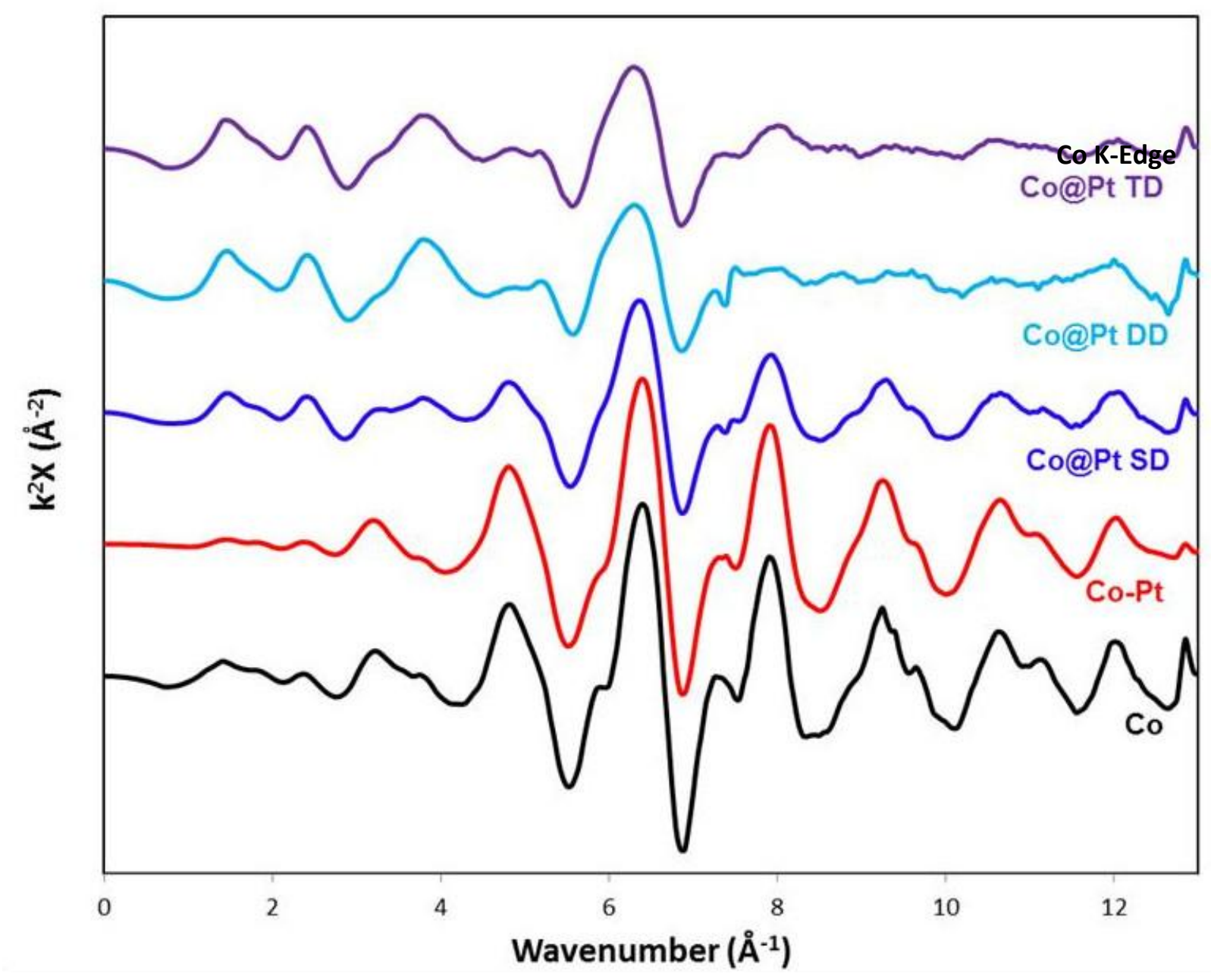

Figure 6:

1

2

3

5 
Figure 7:

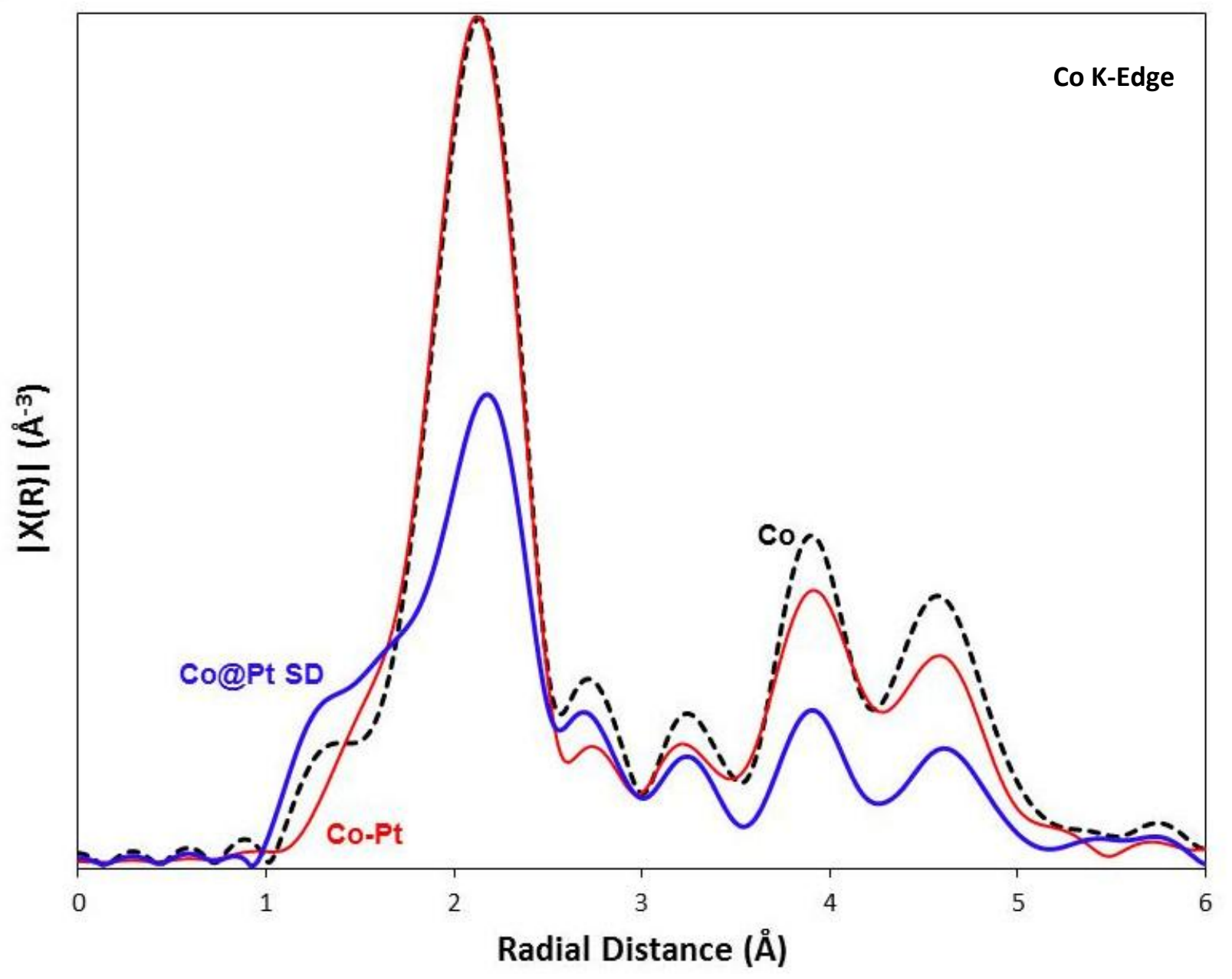


Figure 8:

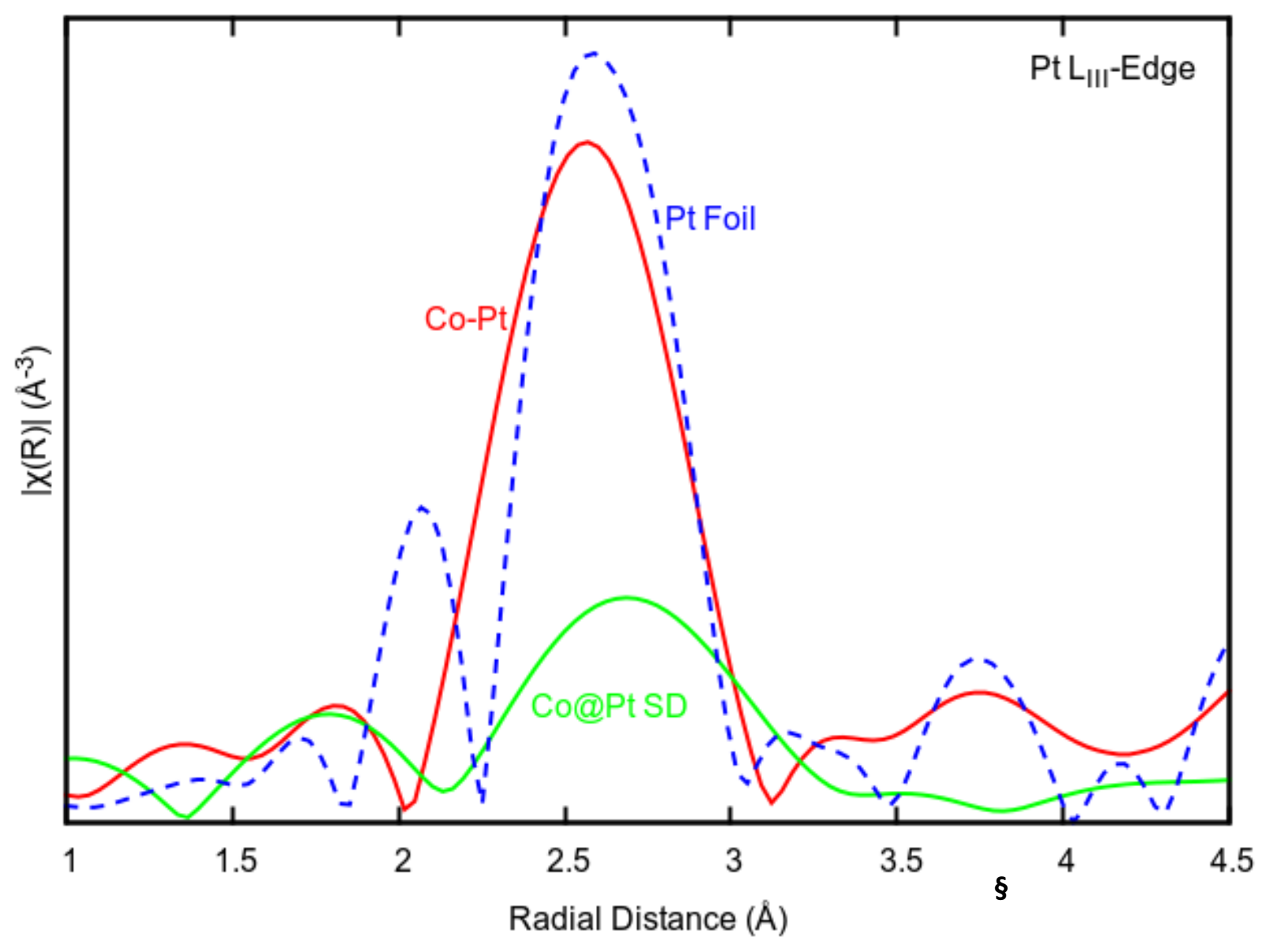


Figure 9:

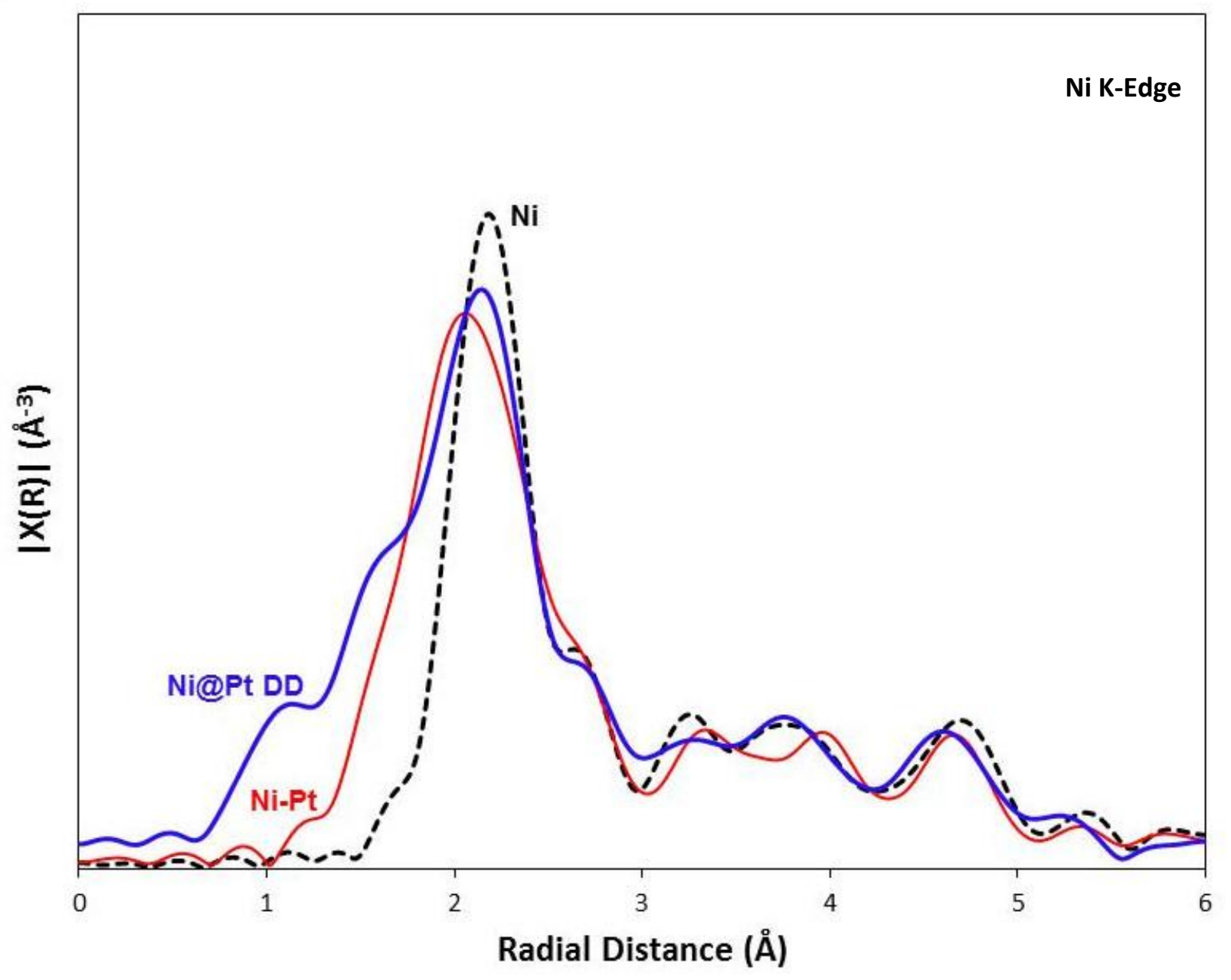


Figure 10:

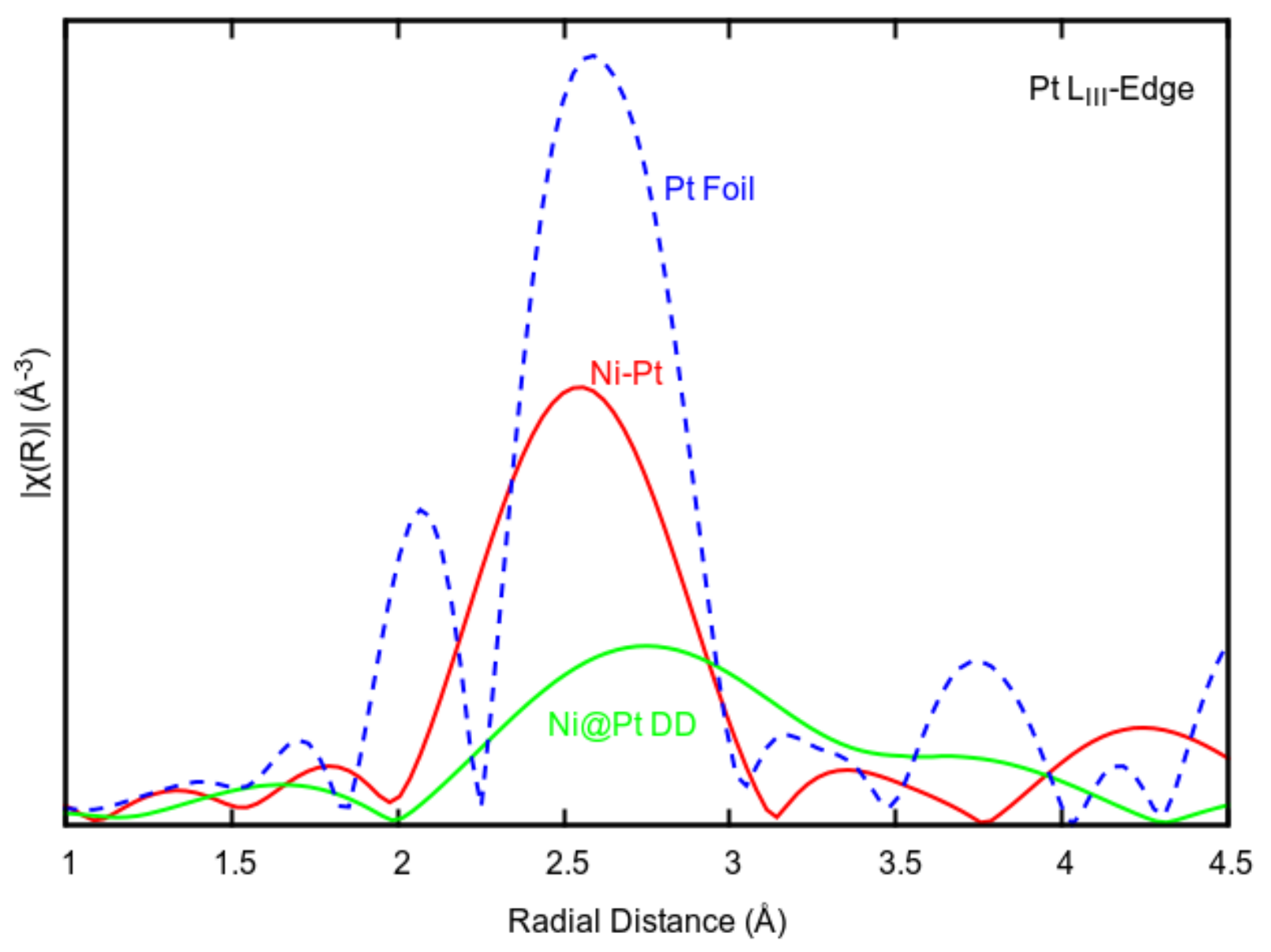


Figure 11:

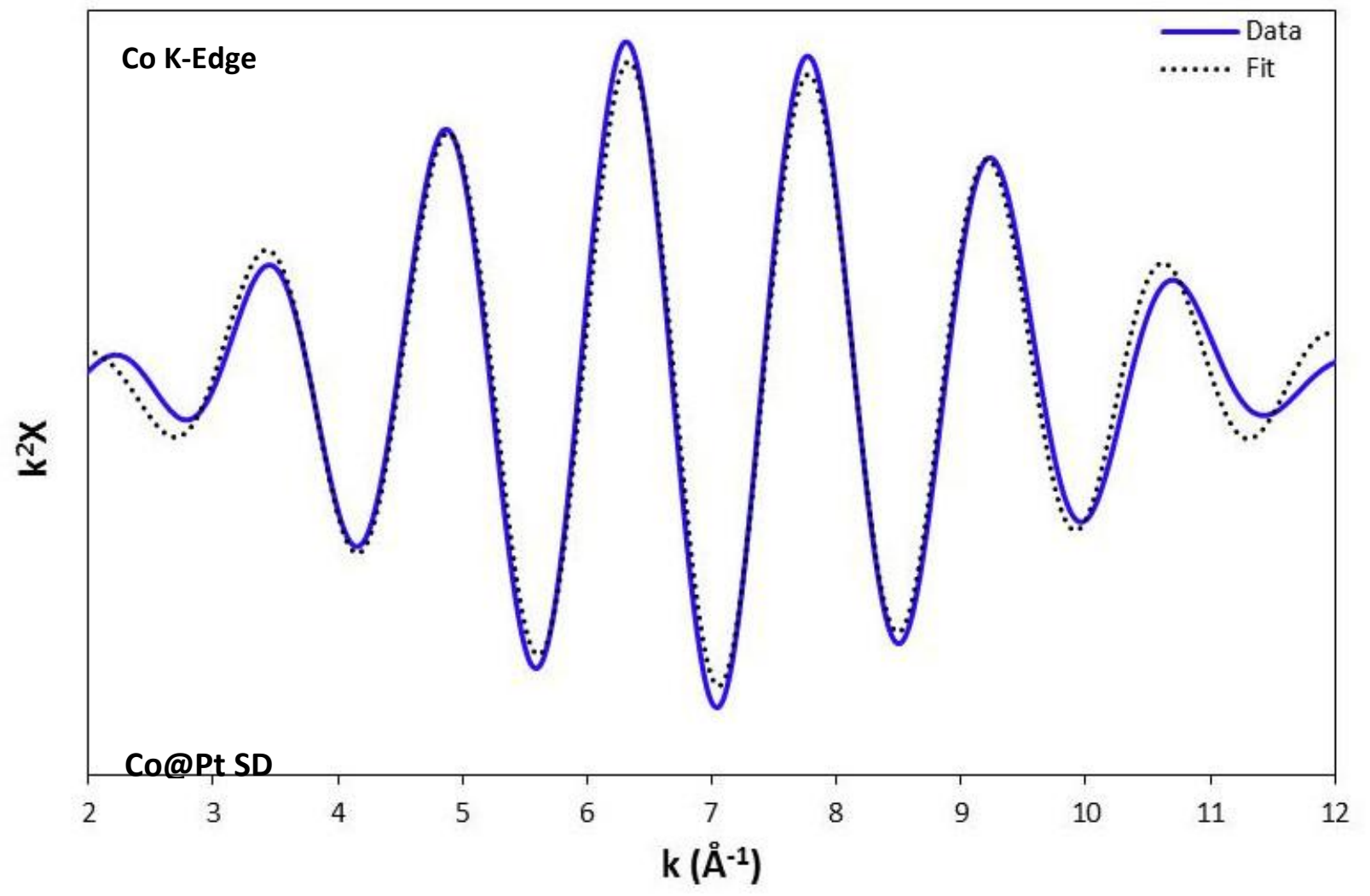


Figure 12:

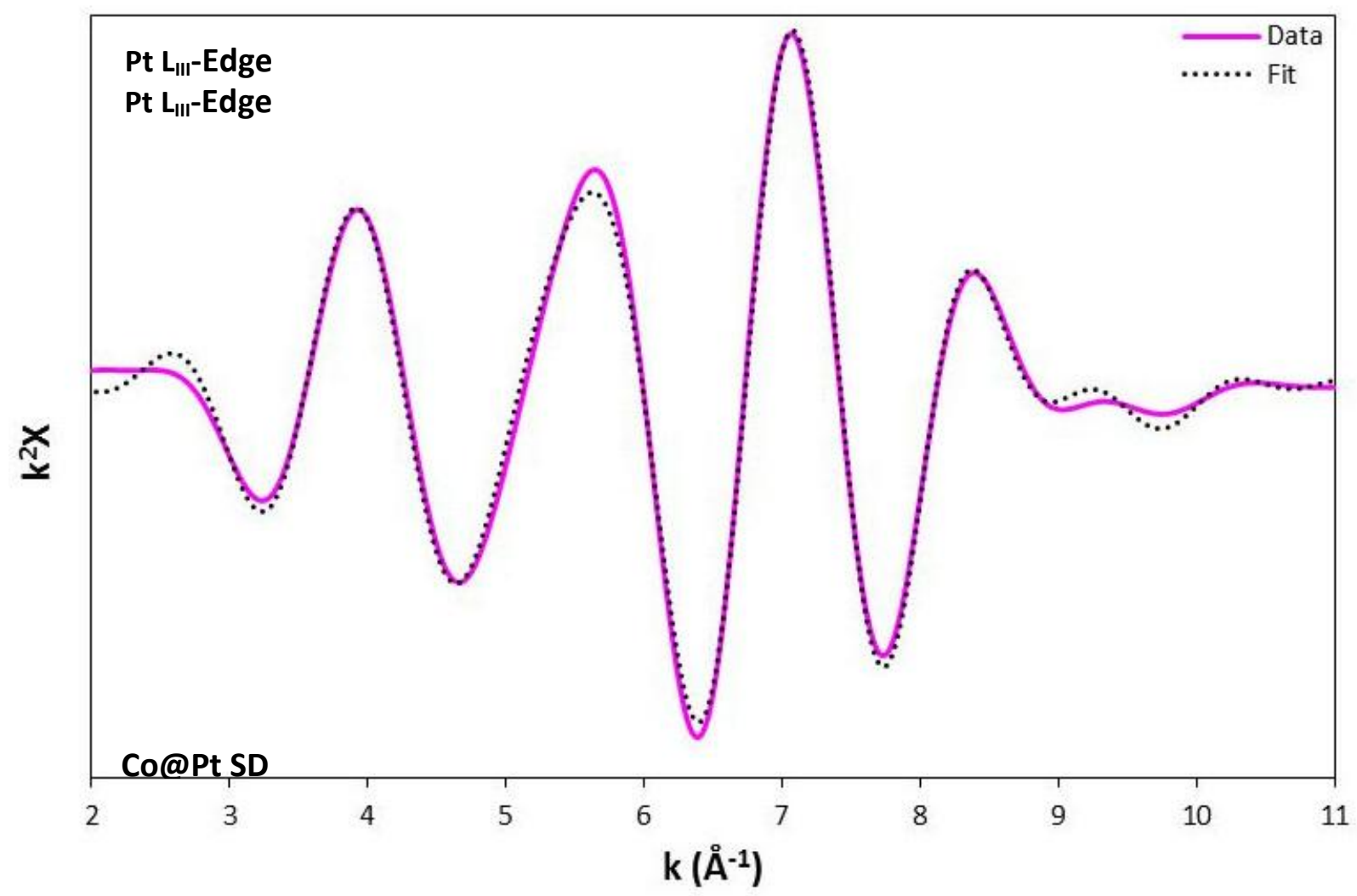


Figure 13:

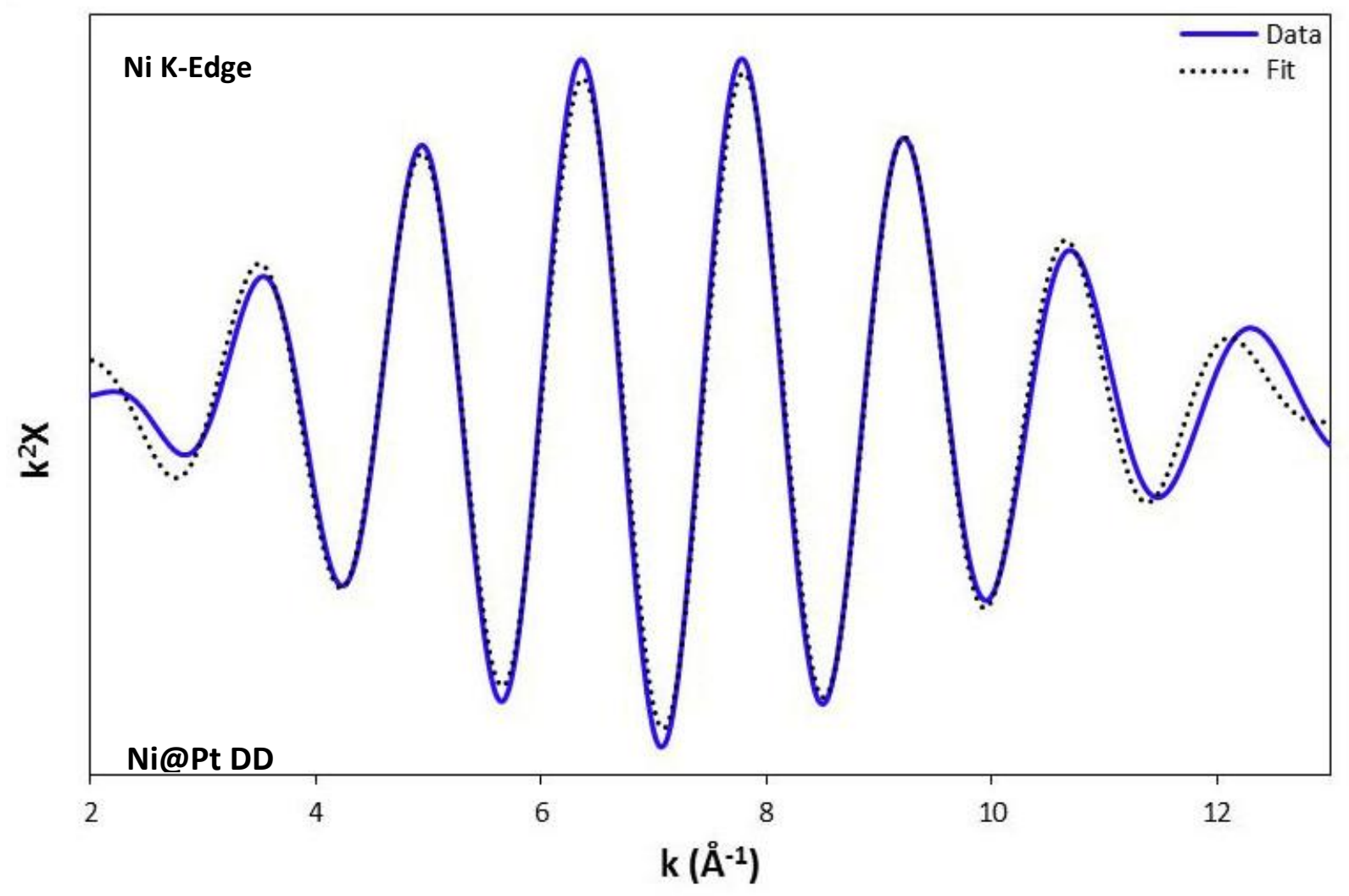


Figure 14:

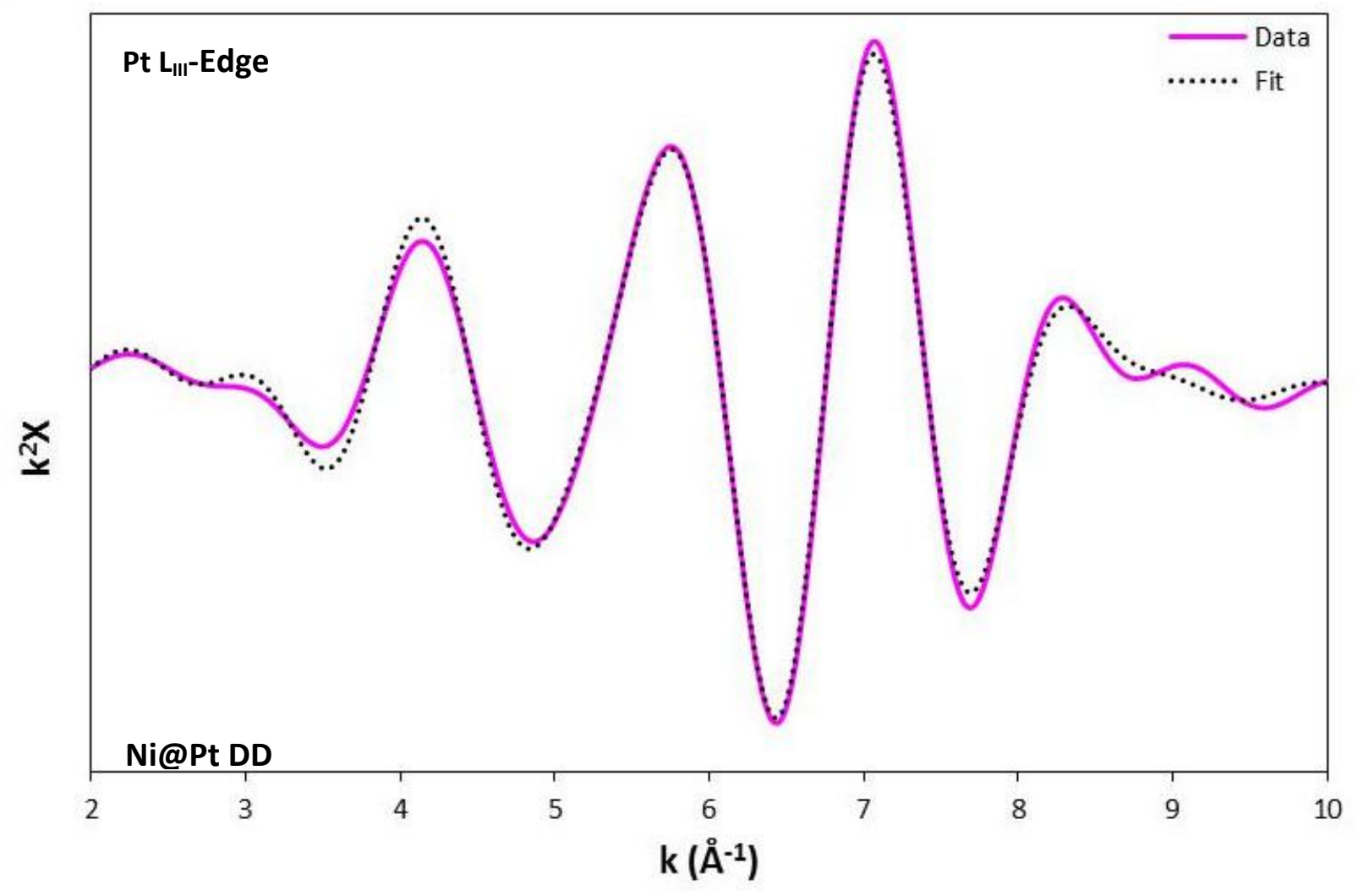


Table 1:

\begin{tabular}{|c|c|c|c|}
\hline Sample & Result wt\% & Metal & Dispersion \\
\hline $\mathrm{Ni} / \mathrm{Al}_{2} \mathrm{O}_{3}$ Parent & $5.02 \%$ & $\mathrm{Ni}$ & $3.9 \%$ \\
\hline Ni@Pt SD & $0.0363 \%$ & $\mathrm{Pt}$ & \\
\hline Ni@Pt DD & $0.0390 \%$ & $\mathrm{Pt}$ & \\
\hline Ni@Pt TD & $0.0482 \%$ & $\mathrm{Pt}$ & \\
\hline \multirow[t]{2}{*}{ Ni-Pt Bimetallic } & $4.51 \%$ & $\mathrm{Ni}$ & $6.4 \%$ \\
\hline & $0.0405 \%$ & $\mathrm{Pt}$ & \\
\hline $\mathrm{Co} / \mathrm{Al}_{2} \mathrm{O}_{3}$ Parent & $4.70 \%$ & Co & $2.8 \%$ \\
\hline Co@Pt SD & $0.0093 \%$ & $\mathrm{Pt}$ & \\
\hline Co@Pt DD & $0.0141 \%$ & $\mathrm{Pt}$ & \\
\hline Co@Pt TD & $0.0134 \%$ & $\mathrm{Pt}$ & \\
\hline \multirow[t]{2}{*}{ Co-Pt Bimetallic } & $5.10 \%$ & Co & $4.3 \%$ \\
\hline & $0.0139 \%$ & $\mathrm{Pt}$ & \\
\hline $\mathrm{Pt} / \mathrm{Al}_{2} \mathrm{O}_{3}$ & $0.4680 \%$ & $\mathrm{Pt}$ & $44.4 \%$ \\
\hline
\end{tabular}


Table 2:

\begin{tabular}{|c|c|c|c|c|c|c|c|}
\hline Sample & $\begin{array}{l}\text { N Pt-M } \\
( \pm 20 \%)\end{array}$ & $\begin{array}{l}\text { R Pt-M A } \\
( \pm 0.01 \AA)\end{array}$ & $\mathbf{M}$ & $\begin{array}{l}\Delta \sigma^{2} \AA^{2} \\
( \pm 10 \%)\end{array}$ & $\delta_{0}^{2}$ (error) & $\begin{array}{l}\Delta \mathrm{E}_{0} \mathrm{eV} \\
\text { (error) }\end{array}$ & $\begin{array}{c}\text { Edge } \\
\text { Energy }(\mathrm{eV})\end{array}$ \\
\hline Pt & 3.7 & 2.76 & $\mathrm{Pt}$ & 0.0063 & $\begin{array}{c}0.84 \\
(0.09)\end{array}$ & $6.3(0.7)$ & 11564 \\
\hline Co-Pt & 10.3 & 2.58 & Co & 0.0127 & $\begin{array}{c}4.93 \\
(0.43)\end{array}$ & $33.3(4.7)$ & 11564 \\
\hline Co@Pt SD & 5.6 & 2.67 & $\mathrm{Pt}$ & 0.0026 & $\begin{array}{c}0.89 \\
(0.24)\end{array}$ & $0.2(1.8)$ & 11565 \\
\hline Co@Pt DD & 7.2 & 2.69 & $\mathrm{Pt}$ & 0.0072 & $\begin{array}{c}0.85 \\
(0.08)\end{array}$ & $1.9(2.8)$ & 11564 \\
\hline Co@Pt TD & 7.7 & 2.79 & $\mathrm{Pt}$ & 0.0051 & $\begin{array}{c}0.85 \\
(0.05)\end{array}$ & $10.0(2.6)$ & 11564 \\
\hline
\end{tabular}


Table 3:

\begin{tabular}{|c|c|c|c|c|c|c|c|}
\hline Sample & $\begin{array}{l}\text { N Pt-M } \\
( \pm 20 \%)\end{array}$ & $\begin{array}{l}\text { R Pt-M Å } \\
( \pm 0.01 \AA)\end{array}$ & $\mathbf{M}$ & $\begin{array}{l}\Delta \sigma^{2} \AA^{2} \\
( \pm 10 \%)\end{array}$ & $\delta_{0}^{2}$ (error) & $\begin{array}{l}\Delta \mathrm{E}_{0} \mathrm{eV} \\
\text { (error) }\end{array}$ & $\begin{array}{c}\text { Edge } \\
\text { Energy }(\mathrm{eV})\end{array}$ \\
\hline Pt & 3.7 & 2.76 & $\mathrm{Pt}$ & 0.0063 & $\begin{array}{c}0.84 \\
(0.09)\end{array}$ & $6.3(0.7)$ & 11564 \\
\hline $\mathrm{Ni}-\mathrm{Pt}$ & 10.0 & 2.54 & $\mathrm{Ni}$ & 0.0134 & $\begin{array}{c}4.52 \\
(0.52)\end{array}$ & $28.8(2.5)$ & 11564 \\
\hline Ni@Pt SD & 6.3 & 2.67 & $\mathrm{Pt}$ & 0.0077 & $\begin{array}{c}0.96 \\
(0.10)\end{array}$ & $-1.1(3.5)$ & 11564 \\
\hline Ni@Pt DD & 7.8 & 2.73 & $\mathrm{Pt}$ & 0.0077 & $\begin{array}{c}0.93 \\
(0.08)\end{array}$ & $11.7(2.6)$ & 11564 \\
\hline Ni@Pt TD & 8.2 & 2.72 & $\mathrm{Pt}$ & 0.0020 & $\begin{array}{c}0.52 \\
(0.04) \\
\end{array}$ & $11.9(0.7)$ & 11564 \\
\hline
\end{tabular}


Table 4:

\begin{tabular}{|c|c|c|c|c|c|c|}
\hline Sample & $\begin{array}{c}\text { N Co- } \\
\text { Co } \\
( \pm 20 \%) \\
\end{array}$ & $\begin{array}{c}\text { R Co-Co } \AA \\
( \pm 0.01 \AA)\end{array}$ & $\begin{array}{l}\Delta \sigma^{2} \AA^{2} \\
( \pm 10 \%)\end{array}$ & $\delta_{0}^{2}$ (error) & $\begin{array}{l}\Delta \mathrm{E}_{0} \mathrm{eV} \\
\text { (error) } \\
\end{array}$ & $\begin{array}{c}\text { Edge } \\
\text { Energy }(\mathrm{eV})\end{array}$ \\
\hline Co & 7.4 & 2.51 & 0.0072 & $\begin{array}{c}0.74 \\
(0.17)\end{array}$ & $-1.3(2.2)$ & 7709 \\
\hline Co-Pt & 7.1 & 2.50 & 0.0067 & $\begin{array}{c}0.74 \\
(0.05)\end{array}$ & $-2.4(1.5)$ & 7712.3 \\
\hline Co@Pt SD & 6.12 & 2.63 & 0.0078 & $\begin{array}{c}0.64 \\
(0.13)\end{array}$ & $1.5(3.6)$ & 7712.6 \\
\hline Co@Pt DD & 6.15 & 2.65 & 0.0205 & $\begin{array}{c}0.99 \\
(0.10)\end{array}$ & 13.5 & 7712.3 \\
\hline Co@Pt TD & 6.07 & 2.64 & 0.0191 & $\begin{array}{c}0.92 \\
(0.12)\end{array}$ & $12.4(2.9)$ & 7712.4 \\
\hline
\end{tabular}


Table 5:

\begin{tabular}{|c|c|c|c|c|c|c|}
\hline Sample & $\begin{array}{l}\text { N Ni-Ni } \\
( \pm 20 \%)\end{array}$ & $\begin{array}{l}\text { R Ni-Ni A } \\
( \pm 0.01 \AA)\end{array}$ & $\begin{array}{l}\Delta \sigma^{2} \AA^{2} \\
( \pm 10 \%)\end{array}$ & $\delta_{0}^{2}$ (error) & $\begin{array}{l}\Delta \mathrm{E}_{0} \mathrm{eV} \\
\text { (error) }\end{array}$ & $\begin{array}{c}\text { Edge } \\
\text { Energy }(\mathrm{eV})\end{array}$ \\
\hline $\mathbf{N i}$ & 8.7 & 2.52 & 0.0100 & $\begin{array}{c}0.68 \\
(0.08)\end{array}$ & $4.3(2.7)$ & 8333 \\
\hline $\mathrm{Ni}-\mathrm{Pt}$ & 7.1 & 2.43 & 0.0091 & $\begin{array}{c}0.74 \\
(0.08)\end{array}$ & $-16.6(1.5)$ & 8334.7 \\
\hline Ni@Pt SD & 8.15 & 2.49 & 0.0110 & $\begin{array}{c}0.77 \\
(0.08)\end{array}$ & $5.4(3.0)$ & 8334.4 \\
\hline Ni@Pt DD & 8.79 & 2.48 & 0.0122 & $\begin{array}{c}0.57 \\
(0.07)\end{array}$ & $-13.6(3.2)$ & 8334.7 \\
\hline Ni@Pt TD & 8.86 & 2.49 & 0.0137 & $\begin{array}{c}1.02 \\
(0.24)\end{array}$ & $-8.1(5.1)$ & 8335.1 \\
\hline
\end{tabular}

